\title{
NONCOMMUTATIVE GEOMETRY BASED ON COMMUTATOR EXPANSIONS
}

\author{
M. Kapranov
}

\section{Contents}

1. NC-complete algebras.

2. NC-schemes.

3. The NC-affine space and Feynman-Maslov operator calculus.

4. Detailed study of algebraic NC-manifolds.

5. Examples of NC-manifolds.

The term "noncommutative geometry" has come to signify a vast framework of ideas directed towards generalization, to noncommutative rings, of the fundamental duality between spaces and (commutative) rings of functions on them. This duality is at the basis of the modern approach to geometry. The main motivation to look for noncommutative generalizations is provided by quantum mechanics where commuting functions are replaced by non-commuting operators.

The study of noncommutative rings by methods and concepts inspired by differential geometry such as (analogs) of differential forms, connections, integrations, Chern classes etc. was pursued very actively and many important results have been obtained, including the discovery of cyclic homology [Co].

From the point of view of algebraic geometry, however, commutative rings by themselves correspond to only a particular class of "spaces", namely affine schemes, and the thrust of the theory is that they can be glued to form more global objects. The construction of such objects in the noncommutative case (and even of any actual geometric objects corresponding to noncommutative rings) has proved to be quite difficult despite many interesting developments [AZ] [Ros] [VV].

The aim of the present paper is to develop an approach to noncommutative algebraic geometry "in the perturbative regime" around ordinary commutative geometry. Let $R$ be a noncommutative algebra (over $\mathbf{C}$ ) and $R_{a b}=R /[R, R]$ be its commutativization. Then we know the geometric object $X_{a b}=\operatorname{Spec}\left(R_{a b}\right)$. The naive aim of noncommutative algebraic geometry would be to associate to the surjection $R \rightarrow R_{a b}$ an embedding of $X_{a b}$ into some "noncommutative space" $X=\operatorname{Spec}(R)$. The essense of our perturbative approach is not to worry about the whole $X$ but concentrate on the formal neighborhood of $X_{a b}$ in $X$. This neighborhood can be described, as in the usual algebro-geometric theory of formal schemes, by equipping $X_{a b}$, a known object, by an appropriate sheaf of noncommutative rings $\mathcal{O}^{N C}$. On the algebraic level, this means that we complete $R$ with respect to the topology, in which iterated commutators, like $\left[a_{1},\left[a_{2}, \ldots,\left[a_{n-1}, a_{n}\right] \ldots\right]\right.$, or $\left[a_{1}, b_{1}\right] \cdot \ldots \cdot\left[a_{n}, b_{n}\right]$, 
are small. Elements of the completion can be thought of as formal commutator series.

Further, ringed spaces of the form $\left(X_{a b}, \mathcal{O}^{N C}\right)$ can be glued together to form more general objects which we call NC-schemes. Any NC-scheme $X$ consists of an ordinary scheme $X_{a b}$ and an appropriate sheaf of rings $\mathcal{O}^{N C}$ on it.

We are especially interested in NC-manifolds, which are NC-schemes $\left(M, \mathcal{O}^{N C}\right)$ where $M$ is a smooth algebraic variety of some dimension $n$, and the completion of $\mathcal{O}^{N C}$ at any $x \in M$ is isomorphic to $\mathbf{C}\left\langle\left\langle x_{1}, \ldots, x_{n}\right\rangle\right\rangle$, the completion of the free associative algebra. This concept globalizes, in a sense, the theory of smooth (quasi-free) noncommutative algebras studied by W. Schelter, J. Cuntz and D. Quillen [Sche][Q].

Given an ordinary manifold $M$, an NC-manifold (thickening) $X$ with $X_{a b}=M$ can be thought of as a sophisticated differential-geometric structure on $M$, leading to additional characteristic classes, e.g., the NC-Atiyah class in $H^{1}\left(M, \Omega_{M}^{2} \otimes T_{M}\right)$, see (4.5). The idea that one can and should seriously develop noncommutative geometry based on free associative algebras (rather than on algebras with relations resembling or relaxing commutativity) was put forward by M. Kontsevich [Ko] in the formal case and I.M. Gelfand and V.S. Retakh [GR] in the affine case. In fact, M. Kontsevich communicated to the author that he was aware of the possibility of a theory such as developed here.

The paper is organized as follows. In Section 1 we study NC-nilpotent algebras, i.e., those in which higher iterated commutators vanish. In particular, we introduce the concept of a $d$-smooth algebra and show (Theorem 1.6.1) that any smooth finitely generated commutative algebra admits a unique (up to a non-canonical isomorphism) $d$-smooth extension. The relation between smooth commutative algebras and $d$-smooth algebras is somewhat similar to the relation between free abelian groups and free $d$-stage nilpotent groups. The class of NC-smooth algebras obtained by passing to the limit $d \rightarrow \infty$, is analogous to the class of free pro-nilpotent groups.

In Section 2 we describe the geometric objects (affine NC-schemes) corresponding to NC-nilpotent algebras as well as well as more global objects obtained by gluing these ones. In particular, we single out the class of algebraic NC-manifolds (whose algebras of functions are NC-smooth) and prove (Theorem 2.3.5) that such manifolds can be identified with appropriate functors on the category of NC-nilpotent algebras.

Section 3 is devoted to a detailed study of the simplest NC-manifold, the noncommutative affine space. It is represented by a certain sheaf $\mathcal{O}^{N C}$ of noncommutative rings on the usual affine space. We give a completely explicit description (Theorem 3.5.3) of this sheaf by using the ideas from the Feynman-Maslov "calculus of ordered operators". The appearance of iterated derivatives in the explicit formulas gives some insight into the differential-geometric meaning of noncommutativization.

In Section 4 we study the problem of constructing a NC-thickeninng of a given ordinary manifold, by homological means. We exhibit a series of cohomological obstructions whose vanishing is necessary and sufficient for the existence of a thickening. These obstructions have a nice interpretation in terms of $A_{\infty}$-structures of J. Stasheff [Sta], the very first 
obstruction being a certain associator.

Finally, in Section 5 we show that several familiar algebraic varieties possess natural NC-thickenings. These include all the classical flag varieties and all the smooth moduli spaces of vector bundles. In particular, for the case of Grassmannians what we get can be seen as a completion, along the commutative points, of the noncommutative Grassmannians of Gelfand-Retakh [GR] which are described as certain explicit functors on the category of skew fields defined in terms of analogs of affine charts.

This research was partially supported by an NSF grant. 


\section{$\S 1$. NC-complete algebras.}

(1.1) The NC-filtration. In this paper the base field is the field $\mathbf{C}$ of complex numbers. In particular, all algebras are $\mathbf{C}$ - algebras.

Let $L$ be a Lie algebra. Its lower central series is the sequence of subalgebras

$$
L_{m}=[L,[L, \ldots,[L, L] \ldots] \quad(m \text { times }), \quad m \geq 1
$$

Thus $L_{1}=L$ and $L_{m}$ is spanned by the expressions $\left[x_{1},\left[x_{2}, \ldots\left[x_{m-1}, x_{m}\right] \ldots\right]\right.$ containing $m-1$ instances of Lie brackets.

Let now $R$ be an associative algebra and $R^{\mathrm{Lie}}=(R,[a, b]=a b-b a)$ be $R$ regarded as a Lie algebra.

(1.1.1) Definition. The NC-filtration of $R$ is the decreasing filtration $\left\{F^{d} R\right\}_{d \geq 0}$ where $F^{d} R$ is the two-sided ideal

$$
F^{d} R=\sum_{m} \sum_{i_{1}+\cdots+i_{m}-m=d} R \cdot R_{i_{1}}^{\mathrm{Lie}} \cdot R \cdot \cdots \cdot R \cdot R_{i_{m}}^{\mathrm{Lie}} \cdot R
$$

Thus $F^{0} R=R$ and $F^{d} R$ is generated by expressions containing $d$ instances of commutator brackets. This filtration was considered by Helton and Howe $[\mathrm{HH}]$ under the name "commutator filtration." Its main property is as follows.

(1.1.2) Proposition. $F$ is a decreasing algebra filtration (i.e., $\left.\left(F^{d_{1}} R\right)\left(F^{d_{2}} R\right) \subset F^{d_{1}+d_{2}} R\right)$ such that the associated graded algebra $\operatorname{gr}_{F}^{\circ} R=\bigoplus F^{d} R / F^{d+1} R$ is commutative. In other words, $\left[F^{d_{1}} R, F^{d_{2}} R\right] \subset F^{d_{1}+d_{2}+1} R$.

The number

$$
\operatorname{ord}_{N C}(f)=\min \left\{d: f \in F^{d} R\right\}
$$

will be called the NC-order of $f \in R$. Note that $\operatorname{gr}_{F}^{0}(R)=R /[R, R]$ is the commutativization of $R$. This algebra will be denoted by $R_{a b}$. Every $\operatorname{gr}_{F}^{i}(R)$ is an $R_{a b}$-module. We have a canonical surjective homomorphism $R \rightarrow R_{a b}$ whose value on $f \in R$ will be denoted $f_{a b}$.

We define the NC-topology on $R$ to be the topology in which the $F^{d} R$ form a basis of neighborhoods of 0 . Recall that a Poisson algebra is a commutative algebra $P$ equipped with an anticommutative binary operation $\{f, g\}$, called the Poisson bracket, which satisfies the Jacobi identy and is a derivation with respect to each argument. A Poisson algebra $P$ will be called graded, if $P=\underset{d \in \mathbf{Z}}{\oplus} P^{d}$ is $\mathbf{Z}$-graded as a vector space, and

$$
P^{d_{1}} \cdot P^{d_{2}} \subset P^{d_{1}+d_{2}}, \quad\left\{P^{d_{1}}, P^{d_{2}}\right\} \subset P^{d_{1}+d_{2}+1} .
$$

Proposition 1.1.2 implies, in a standard way, the following. 
(1.1.4) Proposition. The algebra $\operatorname{gr}_{F}^{\bullet}(R)$ has a natural structure of a graded Poisson algebra.

(1.1.5) Definition. (a) An associative algebra $R$ is called NC-nilpotent of degree $d$ (resp. $N C$-nilpotent), if $F^{d+1} R=0$ (resp. $F^{i} R=0$ for $i \gg 0$ ).

(b) For an associative algebra $R$ its $N C$-completion is the algebra

$$
R_{\llbracket a b \rrbracket}=\lim _{\leftarrow} R / F^{d} R
$$

(c) $R$ is called NC-complete, if the natural morphism $R \rightarrow R_{\llbracket a b \rrbracket}$ is an isomorphism.

(1.1.6) Proposition. The following are equivalent:

(i) $R$ is NC-complete.

(ii) $R$ is Hausdorff and complete with respect to the NC-topology.

(iii) $R$ is an inverse limit of $\mathrm{NC}$-nilpotent algebras.

(1.2) Central extensions. Let $R$ be an associative albebra, $M$ be an $R$-bimodule. We say that $M$ is central, if $a m=m a$ for any $a \in R, m \in M$.

(1.2.1) Proposition. A central $R$-bimodule is the same as a module over $R_{a b}$.

(1.2.2) Definition. Let $R$ be an associative algebra. An Abelian extension of $R$ is an exact sequence of algebras

$$
0 \rightarrow I \rightarrow R^{\prime} \rightarrow R \rightarrow 0
$$

where the ideal $I$ satisfies $I^{2}=0$. A central extension is an Abelian extension such that $I$ lies in the center of $R^{\prime}$.

Thus, in an Abelian extension, the only nontrivial structure on $I$ is that of an $R$ bimodule. For a central extension, $I$ is a central $R$-bimodule.

(1.2.3) Proposition. (a) NC-nilpotent algebras are precisely algebras which can be obtained as iterated central extensions of commutative algebras.

(b) Any surjection $R_{1} \rightarrow R_{2}$ of NC-nilpotent algebras whose kernel is a nilpotent ideal, can be decomposed into a sequence of central extensions.

Given an algebra $R$ and an $R$-bimodule $M$, we will denote by $R \oplus M$ the trivial Abelian extension of $R$ by $M$, which is the direct sum with the product given by

$$
\left(a_{1}, m_{1}\right)\left(a_{2}, m_{2}\right)=\left(a_{1} a_{2}, m_{2} a_{2}+a_{1} m_{2}\right) .
$$

(1.2.5) Proposition. Let

$$
0 \rightarrow I \rightarrow R^{\prime} \stackrel{p}{\rightarrow} R \rightarrow 0
$$

be a central extension. Then:

(a) The group of automorphisms of the extension identical on $I, R$ is Abelian and is identified with $\operatorname{Der}\left(R_{a b}, I\right)$, the module of $I$-valued derivations.

(b) The fiber product $R^{\prime} \times{ }_{R} R^{\prime}$ is identified with

$$
R^{\prime} \underset{R_{a b}}{\times}\left(R_{a b} \oplus I\right) .
$$


Proof; (a) Given any Abelian (not necessarily central) extension, the group of its automorphisms as above is identified with

$$
\operatorname{Der}(R, I)=\{D: R \rightarrow I \mid D(a b)=a D(b)+D(a) b\}
$$

Indeed, for such an automorphism $g$ the map $g-1: R^{\prime} \rightarrow R^{\prime}$ takes values in $I$ and vanishes on $I$, so descends to $D: R \rightarrow I$, which lies in $\operatorname{Der}(R, I)$.

Now, since $I$ is central, one verifies by the Leibniz rule that $D(a b c d)=D(a c b d)$, so $D$ descends to a derivation $R_{a b} \rightarrow I$. This shows that $\operatorname{Der}(R, I)=\operatorname{Der}\left(R_{a b}, I\right)$.

(b) Define

$$
\varphi: R^{\prime} \underset{R}{\times} R^{\prime} \rightarrow R^{\prime} \underset{R_{a b}}{\times}\left(R_{a b} \oplus I\right), \quad(x, y) \mapsto\left(x, x_{a b}+y-x\right) .
$$

It is clear that $\varphi$ is bijective. The fact that $\varphi$ is a homomorphism follows because $I^{2}=0$ and the $R$-action on $I$ factors through $R_{a b}$.

(1.2.6) Proposition. Let $p: R^{\prime} \rightarrow R$ be a central extension as in (1.2.5) and $g: R^{\prime} \rightarrow R^{\prime}$ an algebra endomorphism such that $p g=p$. If $p_{*}: R_{a b}^{\prime} \rightarrow R_{a b}$ is an isomorphism, then $\left.g\right|_{I}=I d$. In particular, $g$ is an isomorphism.

Proof: Consider the homomorphism

$$
\Psi: R^{\prime} \stackrel{(I d, g)}{\rightarrow} R^{\prime} \underset{R}{\times} R^{\prime} \stackrel{\varphi}{\simeq} R^{\prime} \underset{R_{a b}}{\times}\left(R_{a b} \oplus I\right) \rightarrow\left(R_{a b} \oplus I\right) .
$$

The algebra $R_{a b} \oplus I$ is commutative, so this homomorphism descends to a homomorphism $\psi: R_{a b}^{\prime} \rightarrow\left(R_{a b} \oplus I\right)$ whose projection to $R_{a b}$ is $p_{*}$. Since $p_{*}$ is an isomorphism, we can view $\psi$ as a homomorphism $R_{a b} \rightarrow R_{a b} \oplus I$ whose composition with the projection to $R_{a b}$ is the identity. The set of such homomorphisms is identified with $\operatorname{Der}\left(R_{a b}, I\right)$. From the nature of the identification $\varphi$ it follows that $g(x)=x+D(p(x))$ for some $D \in \operatorname{Der}\left(R_{a b}, I\right)$, so $g$ is an automorphism of the form described in (1.2.5)(a).

(1.2.7) Corollary. Let $R_{i}^{\prime} \stackrel{p_{i}}{\rightarrow} R, i=1,2$, be two central extensions of $R$ with $p_{i *}: R_{i, a b}^{\prime} \rightarrow$ $R_{a b}$ isomorphisms. Suppose there are homomorphisms $f: R_{1}^{\prime} \rightarrow R_{2}^{\prime}, g: R_{2}^{\prime} \rightarrow R_{1}^{\prime}$ compatible with the $p_{i}$. Then $R_{1}^{\prime}$ is isomorphic to $R_{2}^{\prime}$. Moreover, the kernels of the $p_{i}$ are identified in a canonical way (i.e., the identification is independent of the choice of $f, g$, provided they exist).

(1.3) Hochschild homology and universal central extensions. Let $R$ be an associative algebra, $I$ be an $R$-bimodule. We denote by $H_{\bullet}(R, I), H^{\bullet}(R, I)$ the Hochschild homolohy and cohomology of $R$ with coefficients in $I$, see $[L]$. The corresponding chain and cochain complexes have the form

$$
C \bullet(R, I)=\left\{I \underset{\mathbf{C}}{\otimes} R^{\otimes n}, n \geq 0\right\}, C^{\bullet}(R, I)=\left\{\operatorname{Hom}_{\mathbf{C}}\left(R^{\otimes n}, I\right), n \geq 0\right\} .
$$

The following is well known. 
(1.3.1) Proposition. $H^{2}(R, I)$ is identified with the set of Abelian extensions (1.2.2) modulo isomorphisms identical on $R$ and $I$.

We now concentrate on the case when $I$ is central.

(1.3.2) Proposition. If $I$ is a central $R$-bimodule, then $C \bullet(R, I), C^{\bullet}(R, I)$ have natural structures of complexes of $R_{a b}$-modules. In particular, $H_{m}(R, I), H^{m}(R, I)$ are $R_{a b^{-}}$ modules.

Proof: Consider, for example, the chain complex. We introduce the $R_{a b}$-module structure in $C_{m}(R, I)=I \underset{\mathrm{C}}{\otimes} R^{\otimes m}$ by viewing $R^{\otimes n}$ as the vector space of multiplicities for the $R_{a b}$-module $I$, i.e.,

$$
a \otimes\left(i \otimes b_{1} \otimes \cdots \otimes b_{m}\right)=a i \otimes b_{1} \otimes \cdots \otimes b_{m}, a \in R_{a b} .
$$

The boundary in $C_{\bullet}(R, I)$ has the form

$$
\begin{aligned}
\partial\left(i \otimes b, \otimes \cdots \otimes b_{m}\right)= & i b_{1} \otimes b_{2} \otimes \cdots \otimes b_{m}+\sum_{\nu=1}^{m-1}(-1)^{\nu} i \otimes \cdots \otimes b_{\nu} b_{\nu+1} \otimes \cdots \otimes b_{m} . \\
& +(-1)^{m} b_{m} i \otimes b_{1} \otimes \cdots \otimes b_{m-1}
\end{aligned}
$$

Because $I$ is central, $\partial$ commutes with the action of $a$. Proposition is proved.

The "universal" example of a central $R$-bimodule is given by $R_{a b}$. The following fact is well known if $R=R_{a b}$ is commutative ([L], Prop. 1.1.10).

(1.3.3) Proposition. Let $R$ be any associative algebra. Then $H_{1}\left(R, R_{a b}\right) \simeq \Omega_{R_{a b}}^{1}$ is the module of Kähler differentials of $R_{a b}$.

Proof: This is a modification of the proof in loc.cit. The relevant part of the Hochschild complex is

$$
\begin{gathered}
R_{a b} \otimes R \otimes R \stackrel{\partial_{2}}{\rightarrow} R_{a b} \otimes R \stackrel{\partial_{1}}{\rightarrow} R_{a b}, \\
\partial_{1}(f \otimes g)=f \cdot G_{a b}-G_{a b} \cdot f=0, \\
\partial_{2}(f \otimes G \otimes H)=f \otimes G H-f G_{a b} \otimes H-H_{a b} f \otimes G .
\end{gathered}
$$

Thus $H_{1}\left(R, R_{a b}\right)=\operatorname{Coker}\left(\partial_{2}\right)$. We now define the maps

$$
\begin{gathered}
\varphi: H_{1}\left(R, R_{a b}\right) \rightarrow \Omega_{R_{a b}}^{1}, \quad f \otimes G \mapsto f \cdot d\left(G_{a b}\right), \\
\psi: \Omega_{R_{a b}}^{1} \rightarrow H_{1}\left(R, R_{a b}\right), \quad f d g \rightarrow f \otimes G \bmod \operatorname{Im}\left(\partial_{2}\right) .
\end{gathered}
$$

Here $G \in R$ is any element such that $G_{a b}=g$. The well-definedness of $\varphi$ is clear. To see that $\psi$ is well defined, we need to prove that for any $A, G_{1}, G_{2}, B \in R, F \in R_{a b}$ we have

$$
f \otimes A G_{1} G_{2} B \equiv f \otimes A G_{2} G_{1} B \quad\left(\bmod \operatorname{Im}\left(\partial_{2}\right)\right) .
$$

To see this, denote $a=A_{a b}, g_{i}=G_{i, a b}$ etc. and find:

$$
f \otimes A G_{1} G_{2} B \equiv f a g_{1} g_{2} \otimes B+b f a g_{1} \otimes G_{2}+g_{1} g_{2} b f \otimes A+g_{2} b f a \otimes G_{1}
$$

. Since $g_{2}, g_{2}$ commute in $R_{a b}$, the right hand side for $f \otimes A G_{2} G_{1} B$ will be the same. Having established the existence of $\varphi$ and $\psi$, it is immediate that they are mutually inverse.

We now note the following universal coefficient formula. 
(1.3.4) Proposition. Let $R$ be any associative algebra, $I$ a central $R$-bimodule. Then there is a spectral sequence

$$
E_{2}^{i j}=\operatorname{Ext}_{R_{a b}}^{j}\left(H_{i}\left(R, R_{a b}\right), I\right) \Rightarrow H^{i+j}(R, I) .
$$

Proof: This follows from the identification of complexes

$$
C^{\bullet}(R, I) \cong \operatorname{Hom}_{R_{a b}}\left(C \cdot\left(R, R_{a b}\right), I\right)
$$

and from the fact that $C_{\bullet}\left(R, R_{a b}\right)$ consists of free $R_{a b}$-modules.

(1.3.5) Corollary. Let $R$ be an associative algebra such that $R_{a b}$ is smooth. Then

$$
H^{2}(R, I)=\operatorname{Hom}_{R_{a b}}\left(H_{2}\left(R, R_{a b}\right), I\right)
$$

for a any central $R$-bimodule $I$. In particular, in this situation we have the tautological class

$$
\tau_{R} \in H^{2}\left(R, H_{2}\left(R, R_{a b}\right)\right)
$$

corresponding to the identity map of $H_{2}\left(R, R_{a b}\right)$.

Proof: Since $R_{a b}$ is smooth, $H_{1}\left(R, R_{a b}\right)=\Omega_{R_{a b}}^{1}$ is projective, so $\operatorname{Ext}_{R_{a b}}^{j}\left(H_{1}\left(R, R_{a b}\right), I\right)=$ $0, j>0$. Also $\operatorname{Ext}_{R_{a b}}^{j}\left(H_{0}\left(R, R_{a b}\right), I\right)=\operatorname{Ext}_{R_{a b}}^{j}\left(R_{a b}, I\right)=0, \quad j>0$. Thus the only nontrivial term $E_{2}^{i j}$ with $i+j=2$ is $\operatorname{Hom}_{R_{a b}}\left(H_{2}\left(R, R_{a b}\right), I\right)$. There are no differentials hitting this term, and the only possible differentials originating from it, are $d_{2}$, with values in $\operatorname{Ext}_{R_{a b}}^{2}\left(H,\left(R, R_{a b}\right), I\right)=0$, and $d_{3}$, with values in a subquotient of $\left.\operatorname{Ext}_{R_{a b}}^{3}\left(H_{0}\left(R, R_{a b}\right)\right), I\right)=$ 0 . This proves our assertion.

(1.3.6) Definition. Let $R$ be an associative algebra such that $R_{a b}$ is smooth. The universal central extension of $R$ is the extension

$$
0 \rightarrow H_{2}\left(R, R_{a b}\right) \rightarrow R^{\tau} \rightarrow R \rightarrow 0
$$

corresponding to the tautological Hochschild class $\tau_{R}$.

Since we have only the class $\tau_{R}$ but not, in general, a distinguished cocycle representing it, we need to make precise in which sense we can speak about "the" universal central extension.

(1.3.7) Proposition. (a) The universal central extension $R^{\tau}$ is defined uniquely up to a (non-canonical) isomorphism identical on $R, H_{2}\left(R, R_{a b}\right)$.

(b) Let $U(R)$ be the category (groupoid) formed by universal central extensions and their isomorphisms as in (a). The $U(R)$ is a gerbe with band $\operatorname{Der}\left(R_{a b}, H_{2}\left(R, R_{a b}\right)\right)$.

Part (b) means that for any two choices $R_{1}^{\tau}$ and $R_{2}^{\tau}$ of the universal central extension, the set of isomorphisms $R_{1}^{\tau} \rightarrow R_{2}^{\tau}$ identical on $R, H_{2}\left(R, R_{a b}\right)$ is a principal homogeneous space over $\operatorname{Der}\left(R_{a b}, H_{2}\left(R, R_{a b}\right)\right)$, and this structure of principal homogeneous space is compatible with the composition of isomorphisms, see [Bry]. This property follows from Proposition 1.2.5(a). 
(1.3.8) Proposition. Let $R$ be as above and

$$
0 \rightarrow J \rightarrow S \rightarrow R \rightarrow 0
$$

be any central extension. Then there is a morphism of extensions $\gamma: R^{\tau} \rightarrow S, \gamma\left(H_{2}\left(R, R_{a b}\right)\right) \subset$ $J$, identical on $R$. This morphism has the property that for any morphism of extensions $\chi: S \rightarrow R^{\tau}, \chi(J) \subset H_{2}\left(R, R_{a b}\right)$, the map $\chi \gamma$ is identical on $H_{2}\left(R, R_{a b}\right)$.

Proof: Let $H=H_{2}\left(R, R_{a b}\right)$ and $c \in H_{2}$ be the class corresponding to $S$. By (1.3.5) we can identify $c$ with a morphism $\tilde{c}: H \rightarrow J$. Such a morphism defines a morphism $\gamma$ of extensions, as claimed. Let now $\chi$ be given. Then $\left.\chi\right|_{J}: J \rightarrow H$ is a morphism of left $R$ bimodules. The fact that $\chi$ is a morphism of extensions means that the image of $c$ under $\left(\left.\chi\right|_{J}\right)_{*}: H^{2}(R, J) \rightarrow H^{2}(R, H)$ is $\tau_{R}$. But this is the same as saying that the composition $\chi \gamma: H \rightarrow H$ is the identity.

(1.3.9) Example. Let $R$ be a smooth commutative algebra. Then $R_{a b}=R$ and $H_{2}(R, R)=\Omega_{R}^{2}$. The class $\tau_{R}$ is represented by the 2-cocycle

$$
c: R \otimes R \rightarrow \Omega_{R}^{2}, \quad c(f, g)=d f \wedge d g .
$$

The universal central extension $R^{\tau}$ is thus explicitly realized as the algebra $R \oplus \Omega_{R}^{2}$ with multiplication

$$
\left(f_{1}, \omega_{1}\right)\left(f_{2}, \omega_{2}\right)=\left(f_{1} f_{2}, f_{1} \omega_{2}+f_{2} \omega_{1}+d f_{1} \wedge d f_{2}\right) .
$$

(1.4) Smooth and $d$-smooth algebras. Let $\mathcal{A l g}$ be the category of all associative algebras and $\mathcal{C}$ om be the subcategory of commutative algebras. Let also $\mathcal{N}_{d}$ be the category of NC-nilpotent algebras of degree $d$ and $\mathcal{N}=\bigcup_{d \geq 0} \mathcal{N}_{d}$. Then we have the inclusions:

$$
\mathcal{C o m}=\mathcal{N}_{0} \subset \mathcal{N}_{1} \subset \cdots \subset \mathcal{N} \subset \mathcal{A l g} .
$$

(1.4.2) Definition. Let $\mathcal{C}$ be one of the categories in (1.4.1). A covariant functor $h: \mathcal{C} \rightarrow$ Sets is called formally smooth, if the following equivalent conditions hold:

(i) For any surjection $p: \Lambda^{\prime} \rightarrow \Lambda$ in $\mathcal{C}$ whose kernel is a nilpotent ideal, the map $h(p): h\left(\Lambda^{\prime}\right) \rightarrow$ $h(\Lambda)$ is surjective.

(ii) For any Abelian extension $p: \Lambda^{\prime} \rightarrow \Lambda$ in $\mathcal{C}$ the map $h(p)$ is surjective.

(1.4.3) Proposition. Let $\mathcal{C}=\mathcal{N}$ or $\mathcal{N}_{d}$ for some $d$. A functor $h: \mathcal{C} \rightarrow$ Sets is formally smooth if an only if $h(p)$ is surjective for any central extension $p: \Lambda^{\prime} \rightarrow \Lambda$ in $\mathcal{C}$.

Proof: See Proposition 1.2.3(b).

For every associative algebra $R$ we have the functor

$$
h^{R}: \mathcal{A l g} \rightarrow \mathcal{S} e t s, \quad \Lambda \mapsto \operatorname{Hom}_{\mathcal{A l g}}(R, \Lambda),
$$

represented by $\Lambda$. If $\mathcal{C}$ is one of the categories above, let $h_{\mathcal{C}}^{R}$ be the restriction of $h^{R}$ to $\mathcal{C}$. The following concept was studied in [Sche] [CQ]. 
(1.4.4) Definition. An associative algebra $R$ is called smooth (or quasi-free), if $h^{R}$ is formally smooth.

We will consider the following version of this concept.

(1.4.5) Definition. Let $R$ be an associative algebra.

(a) For $d \geq 0$ we say that $R$ is $d$-smooth, if $R$ is finitely generated, $R \in \mathcal{N}_{d}$ and $h_{\mathcal{N}_{d}}^{R}$ is formally smooth.

(b) We say that $R$ is $N C$-smooth, if $R$ is $N C$-complete and $R / F^{d+1} R$ is $d$-smooth for each $d$.

For example, a 0-smooth algebra is the same as a smooth commutative algebra, i.e. the coordinate algebra of a smooth affine algebraic variety (Grothendieck's criterion). If $R$ is a $d$-smooth or NC-smooth algebra, we denote by $\operatorname{dim}(R)$ the dimension of the algebraic variety $\operatorname{Spec}\left(R_{a b}\right)$. It is clear that for a $d$-smooth $R$ each $R / F^{i+1} R, i \leq d$ is $i$-smooth. Given a smooth commutative algebra $A$, we call a $d$-smooth thickening of $A$ a $d$-smooth algebra $R$ together with an isomorphism $R_{a b} \rightarrow A$.

(1.4.6) Proposition. Let $R$ be a finitely generated quasi-free algebra. Then $R / F^{d+1} R$ is $d$-smooth for any $d \geq 0$, and $R_{\llbracket a b \rrbracket}$ is NC-smooth.

Proof: Follows from $h_{\mathcal{N}_{d}}^{R}=h_{\mathcal{N}_{d}}^{R / F^{d+1}}$.

Given two algebras $R, S$, we denote by $R * S$ their free product, and by $R \hat{*} S=$ $(R * S)_{\llbracket a b \rrbracket}$ its NC-completion.

(1.4.7) Proposition. (a) If $R, S$ are $d$-smooth, then so is $(R * S) / F^{d+1}(R * S)$.

(b) If $R, S$ are $N C$-smooth, then so is $R \hat{*} S$.

Proof. (a) $(R * S) / F^{d+1}(R * S)$ is the categorical product of $R$ and $S$ in $\mathcal{N}_{d}$. Thus the functor $h_{\mathcal{N}_{d}}^{(R * S) / F^{d+1}}$ takes $\Lambda \mapsto h_{N-d}^{R}(\Lambda) \times h_{\mathcal{N}_{d}}^{S}(\Lambda)$ and it is formally smooth.

(b) Follows form (a) and from the identification

$$
(R * S) / F^{d+1}(R * S) \simeq\left(\left(R / R^{d+1} R\right) *\left(S / F^{d+1} S\right)\right) / F^{d+1} .
$$

(1.4.8) Corollary. If $R_{1}, \ldots, R_{n}$ are coordinate algebras of smooth affine algebraic curves, then $R_{1} \hat{*} \cdots \hat{*} R_{n}$ is NC-smooth.

Proof: Follows from the fact that each $R_{i}$ is quasi-free.

(1.5) Completions of $d$-smooth algebras. Let $\mathbf{C}\left\langle x_{1}, \ldots, x_{n}\right\rangle=\mathbf{C}\left[x_{1}\right] * \cdots * \mathbf{C}\left[x_{n}\right]$ be the free associative algebra on generators $x_{1}, \ldots, x_{n}$. We can view its elements as noncommutative polynomials. Denote by $\mathbf{m} \subset \mathbf{C}\left\langle x_{1}, \ldots, x_{n}\right\rangle$ the two-sided ideal generated by the $x_{i}$. The $\mathbf{m}$-adic completion of $\mathbf{C}\left\langle x_{1}, \ldots, x_{n}\right\rangle$ will be denoted $\mathbf{C}\left\langle\left\langle x_{1}, \ldots, x_{n}\right\rangle\right\rangle$ and called the ring of noncommutative power series. This is a local ring whose maximal ideal $\hat{\mathbf{m}}$ is generated by the $x_{i}$. 
Suppose we have an associative algebra $R$, and $x \in \operatorname{Spec}\left(R_{a b}\right)$ is a $\mathbf{C}$-point. Let $\mathbf{m}_{x, a b} \subset R_{a b}$ be the corresponding maximal ideal, and $\mathbf{m}_{x}=\pi^{-1}\left(m_{x, a b}\right) \subset R$ be its preimage. The $\mathbf{m}_{x}$-adic completion of $R$ will be denote by $\hat{R}_{x}$. Its maximal ideal will be denoted by $\hat{\mathbf{m}}_{x}$.

(1.5.1) Proposition. (a) Let $R$ be $d$-smooth, $\operatorname{dim}(R)=n$, and $x \in \operatorname{Spec}\left(R_{a b}\right)(\mathbf{C})$. Then $\hat{R}_{x} \simeq \mathbf{C}\left\langle\left\langle x_{1}, \ldots, x_{n}\right\rangle\right\rangle / F^{d+1}$.

(b) Let $R$ be $N C$-smooth, $\operatorname{dim} R=n$ and $x$ be as above. Then $\hat{R}_{x} \simeq \mathbf{C}\left\langle\left\langle x_{1}, \ldots, x_{n}\right\rangle\right\rangle$.

Proof: (a) $\mathbf{C}\left\langle x_{1}, \ldots, x_{n}\right\rangle / F^{d+1}$ is the free algebra in $\mathcal{N}_{d}$ generated by $x_{x}, \ldots, x_{n}$, and $\mathbf{C}\left\langle\left\langle x_{1}, \ldots, x_{n}\right\rangle\right\rangle / F^{d+1}$ is its completion. If $\operatorname{dim}(R)=n$, then $\mathbf{m}_{x} / \mathbf{m}_{x}^{2}$ is an $n$-dimensional vector space. Our statement is proved in the same way as the formal tubular neighborhood theorem of Cuntz and Quillen ([CQ], $\S 6$, Th. 2). In fact, it is a version of that theorem but for algebras satisfying the polynomial identities expressed by $F^{d+1}=0$. Part (b) follows from (a).

(1.5.2) Corollary. Let $R, x, n$ be as in (1.5.1). Then:

(a) If $R$ is $d$-smooth and $i \leq d$, then $R / \mathbf{m}_{x}^{i+1} \simeq \mathbf{C}\left\langle x_{1}, \ldots, x_{n}\right\rangle / \mathbf{m}^{i+1}$.

(b) If $R$ is $N C$-smooth and $i \geq 0$, then

$$
R / \mathbf{m}_{x}^{i+1} \simeq \mathbf{C}\left\langle x_{1}, \ldots, x_{n}\right\rangle / \mathbf{m}^{i+1}
$$

In any of these cases we have a natural isomorphism $\mathbf{m}_{x}^{i} / \mathbf{m}_{x}^{i+1} \simeq\left(\mathbf{m}_{x} / \mathbf{m}_{x}^{2}\right)^{\otimes i}$.

(1.6) Existence and uniqueness of thickenings. We now formulate the main result of this section.

(1.6.1) Theorem. Let $A$ be a finitely generated smooth commutative algebra. Then:

(a) For any $d \geq 0 A$ possesses a $d$-smooth thickening $R \rightarrow A$ (with $R_{a b} \rightarrow A$ being an isomorphism). This thickening is unique up to an isomorphism identical on $A$.

(b) Similar statement for NC-smooth thickenings.

Proof: Only (a) needs to be proved, since (b) is a formal consequence. So we start with the proof of uniqueness in (a). Let $R^{\prime}, R^{\prime \prime}$ be two $d$-smooth thickenings of $A$. By induction we may assume that $R^{\prime} / F^{d} R^{\prime}$ and $R^{\prime \prime} / F^{d} R^{\prime \prime}$ are isomorphic and identify them both with the same $(d-1)$-smooth thickening $R_{d-1}$, as in the following diagram:

$$
\begin{aligned}
& 0 \rightarrow I^{\prime} \rightarrow R^{\prime} \rightarrow R_{d-1} \rightarrow 0 \\
& \downarrow \uparrow \quad f \downarrow \uparrow g \quad \downarrow \mathrm{Id} \\
& 0 \rightarrow I^{\prime \prime} \rightarrow R^{\prime \prime} \rightarrow R_{d-1} \rightarrow 0
\end{aligned}
$$

Because both $R^{\prime}, R^{\prime \prime}$ are $d$-smooth there exist morphisms of extensions $f, g$ as shown. Since $R_{a b}^{\prime}=R_{a b}^{\prime \prime}=\left(R_{d-1}\right)_{a b}$, we find that $f$ and $g$ are isomorphisms by Corollary 1.2.7.

We now prove the existence. It is enough to show the following more precise fact. 
(1.6.2) Proposition. Let $R$ be a $d$-smooth algebra and $R^{\tau}$ be its universal central extension. Then $R^{\tau}$ is $(d+1)$-smooth.

It is enough to prove that any surjection $\pi_{d+1}: S_{d+1} \rightarrow R^{\tau}$ with nilpotent kernel and $S_{d+1} \in N_{d+1}$, splits, i.e., admits $\sigma_{d+1}: R^{\tau} \rightarrow S_{d+1}$ with $\pi_{d+1} \sigma_{d+1}=$ Id. Let $S_{d}=$ $S_{d+1} / F^{d+1} S_{d+1}$ and $q: S_{d+1} \rightarrow S_{d}$ be the natural projection. Then $S_{d} \in \mathcal{N}_{d}$. We will now gradually construct the following diagram:

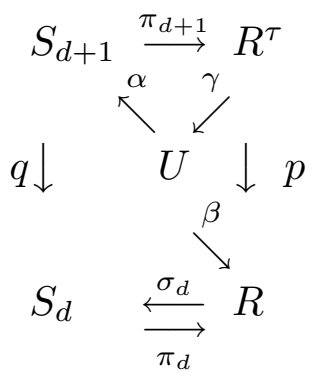

Here $\pi_{d}$ is the surjection induced by $\pi_{d}$ (it exists becaue $R \in \mathcal{N}_{d}$ ). Since $R$ is $d$-smoooth, we have $\sigma_{d}$ with $\pi_{d} \sigma_{d}=\mathrm{Id}$. Let $U$ be the fiber product of $S_{d+1}$ and $R$ over $S_{d}$ with respect to $q, \sigma_{d}$, and $\alpha, \beta$ its natural projections. Let $I=\operatorname{Ker}(q)$ and $H=H_{2}\left(R, R_{a b}\right)=\operatorname{Ker}(p)$. Then $\beta: U \rightarrow R$ is a central extension with kernel $I$ (since $q: S_{d+1} \rightarrow S_{d}$ is). Further, $\pi_{d+1} \alpha: U \rightarrow R^{\tau}$ is a morphism of central extensions because $p \pi_{d+1} \alpha=\pi_{d} q \alpha=\pi_{d} \sigma_{d} \beta=\beta$. Now, by Proposition 1.3.8, there exist a morphism of extensions $j: R^{\tau} \rightarrow U$. Define $\rho_{d+1}=\alpha j: R^{\tau} \rightarrow S_{d+1}$.

(1.6.3) Lemma. $\pi_{d+1} \rho_{d+1}: R^{\tau} \rightarrow R^{\tau}$ is an isomorphism.

Given the lemma, in order to construct $\sigma_{d+1}$, we need just to compose $\rho_{d+1}$ with the inverse of $\pi_{d+1} \rho_{d+1}$.

Proof of the lemma: First, notice that the endomorphism of $R$ induced by $\pi_{d+1} \rho_{d+1}$, is the identity, i.e. $p \pi_{d+1} \rho_{d+1}=p$. Indeed,

$$
p \pi_{d+1} \rho_{d+1}=p \pi_{d+1} \alpha \gamma=\pi_{d} q \alpha \gamma=\pi_{d} \sigma_{d} \beta \gamma=\beta \gamma=p .
$$

Further, the endomorphism of $H=\operatorname{Ker}(p)$ induced by $\pi_{d+1} \rho_{d+1}$ is the identity by Proposition 1.3.8. So $\pi_{d+1} \rho_{d+1}$ is an isomorphism.

This completes the proof of Proposition 1.6.2 and Theorem 1.6.1.

(1.6.4) Remarks. Thus for a smooth finitely generated commutative algebra $A$ the unique $d$-smooth thickening can be constructed by repeatedly taking the universal central extension, starting from $A$. One can compare this with a similar situation in group theory.

If $G$ is a group whose Abelianization $G_{a b}=H_{1}(G, \mathbf{Z})$ is free, then the universal coefficients formula gives a tautological class $\tau_{G} \in H^{2}\left(G, H_{2}(G, \mathbf{Z})\right)$ and we can form the "universal" central extension with kernel $H_{2}(G, \mathbf{Z})$. Taking a free Abelian group $A$ with $n$ generators and applying this construction $d$ times, we get the free $(d+1)$-stage nilpotent group on $n$ generators. See $[\mathrm{Ev}][\mathrm{BE}]$ where this folklore fact is implicit. As in (1.3.9), 
the very first universal central extension (with kernel $\bigwedge^{2} A$ ) is functorial in $A$, but the subsequent ones are not. 


\section{$\S 2$. NC-schemes.}

(2.1) Localization of NC-nilpotent algebras. We start with the following obvious remark.

(2.1.1) Proposition. If $R$ is an $N C$-complete algebra, $A=\left\|a_{i j}\right\| \in \operatorname{Mat}_{m}(R)$ be a square matrix such that $A_{a b}=\left\|\left(a_{i j}\right)_{a b}\right\| \in \operatorname{Mat}_{m}\left(R_{a b}\right)$ is invertible, then $A$ is invertible.

Proof: Let $B \in \operatorname{Mat}_{m}(R)$ be such that $B_{a b}=A_{a b}^{-1}$, i.e., $U=A B-1 \in \operatorname{Mat}_{m}\left(F^{1} R\right)$. Now we find $A^{-1}=\sum_{d=0}^{\infty} B \cdot(-1)^{d} \cdot U^{d}$, where the series converges in the topology on $\operatorname{Mat}_{m}(R)$ induced by the NC-topology on $R$.

(2.1.2) Corollary. If $R$ is $N C$-complete and $R_{a b}$ is local, then $R$ is local.

We now recall the framework of Ore localization [Ste].

(2.1.3) Definition. Let $R$ be an associative algebra. A multiplicative subset $S \subset R-\{0\}$ is said to satisfy the Ore conditions, if the following hold:

(OL1) For any $a \in R, s \in S$ there are $b \in R, u \in S$ such that $u a=b s$.

(OL2) If as $=0$ with $a \in R, s \in S$, then $t a=0$ for some $t \in S$.

(OR1) For any $b \in R, u \in S$ there are $a \in R, s \in S$ such that $u a=b s$.

(OR2) If $s a=0$ with $a \in R, s \in S$, then at $=0$ for some $t \in S$.

If $S$ satisfies the Ore conditions one defines the algebra of fractions $R\left[S^{-1}\right]$ as the set of equivalence classes of, say, right fractions $u^{-1} b$, see [Ste], Ch. II. This algebra has the following properties.

(2.1.4) Proposition. (a) There exist a natural homomorphism $c: R \rightarrow R\left[S^{-1}\right]$ taking any $s \in S$ into an invertible element.

(b) For any algebra homomorphism $\varphi: R \rightarrow R^{\prime}$ such that $\varphi(s)$ is invertible for any $s \in S$, there is a unique homomorphism $\bar{\varphi}: R\left[S^{-1}\right] \rightarrow R$ such that $\varphi=\bar{\varphi} c$.

(c) $R\left[S^{-1}\right]$ is flat as a left and as a right $R$-module.

(d) $c(a)=0$ if and only if as $=0$ for some $s \in S$.

All these properties can be found in [Ste], Ch. II. More precisely, (c) is Prop. 3.5 of loc. cit., (b) is Prop. 1.1 and (a),(d) are properties F1,F3 which define the abstract concept of a ring of fractions (of which the Ore construction proves the existence).

(2.1.5) Proposition. Let $R$ be $N C$-nilpotent, $\pi: R \rightarrow R_{a b}$ the Abelianization map and $\bar{S} \subset R_{a b}-\{0\}$ be any multiplicative subset. Then $S=\pi^{-1}(\bar{S})$ satisfies the Ore conditions.

Proof: (OL1) For every $n \geq 0$ we have the identity

$$
s^{n+1} a=\left(\sum_{i=0}^{n} s^{n-i} a d(s)^{i}(a)\right) s+a d(s)^{n+1}(a) .
$$


If $F^{n+1} R=0$, the last term on the right vanishes. So taking

$$
u=s^{n+1}, b=\sum_{i=0}^{n} s^{n-i} a d(s)^{i}(a),
$$

we get $u a=b s$.

(OL2) If $a s=0$, then we find inductively:

$$
s a=[s, a], s^{2} a=[s, s a]=[s,[s, a]], \ldots, s^{n} a=a d(s)^{n} a .
$$

Thus if $F^{n} R=0$, then $s^{n} a=0$. The proof of (OR1-2) is similar.

(2.1.6) Theorem. Let $R$ be NC-nilpotent, $\bar{S} \subset R_{a b}-\{0\}$ a multiplicative subset, and $S=\pi^{-1}(\bar{S}) \subset R$ its preimage. Then $R\left[S^{-1}\right]$ is NC-nilpotent and

$$
\operatorname{gr}_{F}^{d}\left(R\left[S^{-1}\right]\right)=\operatorname{gr}_{F}^{d}(R)\left[\bar{S}^{-1}\right],
$$

where on the right we have the usual module of fractions of the $R_{a b}$-module $\operatorname{gr}_{F}^{d}(R)$.

The proof is based on the following fact.

(2.1.7) Proposition. We have $F^{d}\left(R\left[S^{-1}\right]\right)=S^{-1} \cdot\left(F^{d} R\right)$.

We first deduce the theorem from the proposition. Because of the properties of modules of fractions with respect to Ore sets ([Ste], Ch. II. §3), we can write $F^{d}\left(R\left[S^{-1}\right]\right)=$ $R\left[S^{-1}\right] \underset{R}{\otimes}\left(F^{d} R\right)$. Further, since $R\left[S^{-1}\right]$ is flat over $R$ by $(2.1 .4)$ (c), we have:

$$
\begin{aligned}
\operatorname{gr}_{F}^{d}\left(R\left[S^{-1}\right]\right) & =R\left[S^{-1}\right] \underset{R}{\otimes} \operatorname{gr}_{F}^{d}(R)=\left(R\left[S^{-1}\right] \underset{R}{\otimes} R_{a b}\right) \underset{R_{a b}}{\otimes} \operatorname{gr}_{F}^{d}(R)= \\
& =R_{a b}\left[\bar{S}^{-1}\right] \underset{R_{a b}}{\otimes} \operatorname{gr}_{F}^{d}(R)=\operatorname{gr}_{F}^{d}(R)\left[\bar{S}^{-1}\right]
\end{aligned} .
$$

We now prove Proposition 2.1.7. It is obviously enough to prove that

$$
\left[s_{0}^{-1} a_{o},\left[s_{1}^{-1} a_{1}, \ldots,\left[s_{d-1}^{-1} a_{d-1}, s_{d}^{-1} a_{d}\right] \ldots\right] \in S^{-1} F^{d} R\right.
$$

for any $s_{i} \in S, a_{i} \in R$. But this is proved by induction, using the identities

$$
\begin{gathered}
{[x y, z]=x[y, z]+[x, z] y, \quad\left[s^{-1}, z\right]=-s^{-1}[s, z] s^{-1},} \\
a s^{-1}=s^{-1} a+s^{-2}[s, a]+s^{-3}[s,[s, a]]+\cdots
\end{gathered}
$$

of which the third one is obtained by iterating the second one (or from (2.1.5.1)). Theorem is proved.

In the sequel we will write $R\left[\bar{S}^{-1}\right]$ for the localization $R\left[\pi^{-1}(\bar{S})^{-1}\right]$, where $R$ is a NC-nilpotent algebra and $\bar{S} \subset R_{a b}$ a multiplicative subset. In particular, we write $R\left[g^{-1}\right]$ for $\left.R\left[\pi^{-1}\left\{g^{i}\right\}\right)^{-1}\right], g \in R_{a b}$. 
(2.1.8) Definition. For an NC-complete algebra $R$ and a multiplicative subset $T \subset R_{a b}$ we define the localization

$$
R \llbracket T^{-1} \rrbracket=\lim _{\leftarrow}\left(R / F^{d+1} R\right)\left[T^{-1}\right] .
$$

Because localization does not commute with inverse limits, not all elements of $R \llbracket T^{-1} \rrbracket$ can be represented as actual fractions.

(2.2) NC-schemes. Let $R$ be an NC-nilpotent algebra. Denote by $X_{a b}=\operatorname{Spec}\left(R_{a b}\right)$ the affine scheme corresponding to the commutative algebra $R_{a b}$. We will now construct a ringed space $X=\operatorname{Spec}(R)=\left(X_{a b}, \mathcal{O}_{X}\right)$ with underlying space $X_{a b}$, i.e., the set of prime ideals in $R_{a b}$. As well known, the basis of topology in $X_{a b}$ is formed by the principal open subsets $D_{g}=\left\{\wp \in \operatorname{Spec}\left(R_{a b}\right): g \notin \wp\right\}$ for $g \in R_{a b}$. We define a presheaf $\tilde{\mathcal{O}}$ on this basis of topology by putting $\tilde{\mathcal{O}}\left(D_{g}\right)=R\left[g^{-1}\right]$, the localization defined in (2.1). Then we set $\mathcal{O}_{X}$ to be the associated sheaf. Explicitly, this means that we first form the stalks

$$
\mathcal{O}_{\wp}:=R_{\wp}=\lim _{\wp \in D_{g}} R\left[g^{-1}\right], \quad \wp \in \operatorname{Spec}\left(R_{a b}\right),
$$

then make $\amalg \mathcal{O}_{\wp}$ into a covering space of $X_{a b}$ in a standard way and define $\mathcal{O}_{X}$ as the sheaf of continuous sections.

(2.2.1) Proposition. (a) Each stalk $\mathcal{O}_{p}$ is a local ring.

(b) We have $\Gamma\left(X_{a b}, \mathcal{O}_{x}\right)=R$.

Proof: (a) Follows from (2.1.2).

(b) We are reduced to the following situation. Given a covering $X_{a b}=\bigcup_{g \in J} D_{g}$, it is required to prove that the Čech complex

$$
R \rightarrow \prod_{g \in J} R\left[g^{-1}\right] \rightarrow \prod_{g_{1}, g_{2} \in J} R\left[g_{1}^{-1}, g_{2}^{-1}\right]
$$

is exact in the middle term. The NC-filtration makes it into a complex of filtered vector spaces with finite filtrations. The associated graded complex is a direct sum of complexes of the form

$$
M \rightarrow \prod_{g \in J} M\left[g^{-1}\right] \rightarrow \prod_{g_{1}, g_{2} \in J} M\left[g_{1}^{-1}, g_{2}^{-1}\right]
$$

where $M=\operatorname{gr}_{F}^{d}(R)$ is an $R_{a b}$-module. The exactness of such a complex is well known. So the original complex is exact as well, proving out statement.

(2.2.2) Definition. Let $R$ be an NC-complete algebra, $X_{a b}=\operatorname{Spec}\left(R_{a b}\right)$. The formal spectrum $\operatorname{Spf}(R)$ is the ringed space $\left(X_{a b}, \mathcal{O}_{X}\right)$ where $\mathcal{O}_{X}$ is the sheaf of topological rings obtained as the inverse limit of the structure sheaves of $\operatorname{Spec}\left(R / F^{d+1} R\right)$.

Ringed spaces of the form $\operatorname{Spf}(R)$ will be called affine NC-schemes. Let $\mathcal{N C}$ be the category of NC-complete algebras. 
(2.2.3) Proposition. The functor $X \mapsto \Gamma\left(X_{a b}, \mathcal{O}_{X}\right)$ establishes an equivalence between the category of affine $\mathrm{NC}$-schemes and the opposite category to $\mathcal{N C}$.

Proof: Follows from (2.2.1).

(2.2.4) Definition. An $N C$-scheme is a ringed space $X=\left(M, \mathcal{O}_{X}\right)$ locally isomorphic to an affine $\mathrm{NC}$-scheme.

Thus every NC-scheme $X$ gives rise to an ordinary scheme $X_{a b}=\left(M, \mathcal{O}_{X, a b}\right)$. Nontrivial examples of NC-schemes will be given in $\S 5$.

(2.2.5) Definition. An NC-scheme $X$ is called $N C$-nilpotent (of degree $d$ ) if $\mathcal{O}_{X}$ is. We say that $X$ is of finite type, if it is $N C$-nilpotent, $X_{a b}$ is a scheme of finite type over $\mathbf{C}$ and the sheaves $\operatorname{gr}_{F}^{d} \mathcal{O}_{X}$ on $X_{a b}$ are coherent.

Recall that $\mathcal{C}$ om $\subset \mathcal{N}$ denote the categories of commutative and NC-nilpotent algebras. An NC-scheme $X$ defines a covariant functor

$$
\tilde{h}_{X}: \mathcal{N C} \rightarrow \mathcal{S e t s}, \quad \tilde{h}_{X}(\Lambda)=\operatorname{Hom}_{\mathrm{NC}-\operatorname{sch}}(\operatorname{Spf}(\Lambda), X) .
$$

Let $h_{X}$ be the restriction of $\tilde{h}_{X}$ to $\mathcal{N}$.

(2.2.7) Proposition. (a) $\tilde{h}_{X}$ commutes with inverse limits in $\mathcal{N C}$.

(b) The restriction of $h_{x}$ to $\mathcal{C}$ om is the functor represented by $X_{a b}$.

(c) The correspondence $X \mapsto h_{X}$ embeds the category of NC-schemes as a full subcategory into the category of covariant functors $\mathcal{N} \rightarrow$ Sets.

Proof: (a) The category $\mathcal{N C}^{o p}$ being a full subcategory of the category of NC-schemes, the statement follows from the general fact that a representable contravariant functor commutes with direct limits. Part (b) is clear. Part (c) follows from (a) and the fact that a NC-complete algebra is an inverse limit of NC-nilpotent ones.

(2.3) Smooth and $d$-smooth NC-schemes. Let $X=\left(M, \mathcal{O}_{X}\right)$ be an NC-scheme. Denote $X^{\leq d}=\left(M, \mathcal{O}_{X} / F^{d+1}\right)$ the $d$ th truncation of $X$. Recall that $\mathcal{N}_{d}$ denotes the category of NC-nilpotent algebras of degree $d$. Let $h_{X}^{\mathcal{N}_{d}}$ be the restriction of the functor $h_{X}$ to $\mathcal{N}_{d}$.

(2.3.1) Definition. (a) An NC-scheme $X$ is called $d$-smooth if $X$ is of finite type, $F^{d+1} \mathcal{O}_{X}=0$ and the functor $h_{X}^{\mathcal{N C}}$ is formally smooth (1.4.2).

(b) $X$ is called smooth, if $X \leq d$ is $d$-smooth for each $d$.

The definitions imply that the underlying scheme $M=X_{a b}$ of a $(d$-) smooth NCscheme $X$ is a smooth algebraic variety. Given a smooth algebraic variety $\mathrm{M}$, we define the category $\operatorname{Th}^{d}(M)$ (resp. $\left.\operatorname{Th}^{\infty}(M)\right)$ whose objects are $d$-smooth (resp. smooth) NCschemes $X$ together with an isomorphism $X_{a b} \rightarrow M$, and morphisms are morphisms of NCschemes identical on $M$. Objects of this category will be called $d$-smooth (resp. smooth) thickenings of $M$. 
(2.3.2) Proposition. Any morphism of $\operatorname{Th}^{d}(M), d \leq \infty$, is an isomorphism.

Proof: Follows from Corollary 1.2.7 and Theorem 1.6.1.

Let a covariant functor $h: \mathcal{N}_{d} \rightarrow \mathcal{S}$ ets be given. For any Cartesian diagram of algebras in $\mathcal{N}_{d}$

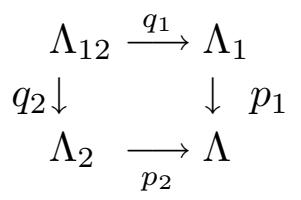

(i.e., $\Lambda_{12} \simeq \Lambda_{1} \underset{\Lambda}{\times} \Lambda_{2}$ ) we have a natural map

$$
j: h\left(\Lambda_{12}\right) \rightarrow h\left(\Lambda_{1}\right) \underset{h(\Lambda)}{\times} h\left(\Lambda_{2}\right) .
$$

The following theorem (cf. [Schl]) will be used to construct many examples of thickenings.

(2.3.5) Theorem. Let $M$ be a smooth algebraic variety and $h_{M}^{\mathcal{C} o m}: \mathcal{C}$ om $\rightarrow$ Sets the corresponding representable functor. Then the category $\operatorname{Th}^{d}(M)\left(\operatorname{resp} . \operatorname{Th}^{\infty}(M)\right)$ is equivalent to the category of formally smooth functors $h: \mathcal{N}_{d} \rightarrow \mathcal{S}$ ets (resp. $\mathcal{N} \rightarrow \mathcal{S}$ ets) such that $\left.h\right|_{\mathcal{C o m}}=h_{M}^{\mathcal{C o m}}$ and satisfying the following left exactness properties:

(1) If, in (2.3.3), $\Lambda_{1}=\Lambda_{2}$ and $p_{1}=p_{2}$ is a central extension, then $j$ is a bijection.

(2) If $\Lambda$ is commutative and $\Lambda_{2}=\Lambda \oplus I$, where $I$ is a $\Lambda$-module, see (1.2.4), then $j$ is a bijection.

Proof: It is enough to consider $d<\infty$ so we assume this. What we really need to do is to prove that any functor satisfying the listed properties, is representable by an NC-scheme, nilpotent of degree $d$. Further, it suffices to prove this when $M$ is affine. Indeed, the affine case being established and given arbitrary $M, h: \mathcal{N}_{d} \rightarrow \mathcal{S}$ ets satisfying the conditions of the theorem, we get a thickening $U^{(d)}$ for any affine open $U \subset M$, and it is defined up to a unique isomorphism. This allows us to glue the $U^{(d)}$ together.

So we assume that $M=\operatorname{Spec}(A)$ is affine. We know that $M$ has a unique, up to an isomorphism, $d$-smooth thickening $X=\operatorname{Spec}(R)$, see Theorem 1.6.1, but we need to identify $h$ with the representable functor $h^{R}: \Lambda \mapsto \operatorname{Hom}(R, \Lambda)$.

Since $h$ is formally smooth and $\pi: R \rightarrow A=R_{a b}$ is a surjection with nilpotent kernel, the identity $\xi_{0} \in h(A)=\operatorname{Hom}(A, A)$, lifts to some $\xi \in h(R)$ which gives a natural transformation $\xi_{*}: h^{R} \rightarrow h$. We are reduced to the following.

(2.3.6) Lemma. $\xi_{*}$ is an isomorphism of functors, i.e., the map

$$
\xi_{*}^{\Lambda}: \operatorname{Hom}(R, \Lambda) \rightarrow h(\Lambda), \quad f \mapsto h(f)(\xi),
$$

is a bijection for any $\Lambda \in \mathcal{N}_{d}$.

Proof: We use the induction the degree of NC-nilpotency of $\Lambda$. So we assume that the statement is true for any $\Lambda \in \mathcal{N}_{d-1}$ and consider a central extension

$$
0 \rightarrow I \rightarrow \Lambda^{\prime} \stackrel{p}{\rightarrow} \Lambda \rightarrow 0, \quad \Lambda \in \mathcal{N}_{d-1} .
$$


Let us prove the bijectivity of $\xi_{*}^{\Lambda^{\prime}}$. By Proposition (1.2.5)(b) and the condition (2) of our theorem, we have a map

$$
h\left(\Lambda^{\prime}\right) \underset{h\left(\Lambda_{a b}\right)}{\times} h\left(\Lambda_{a b} \oplus I\right) \rightarrow h\left(\Lambda^{\prime}\right) \underset{h(\Lambda)}{\times} h\left(\Lambda^{\prime}\right) .
$$

For every $\eta \in h(\Lambda)$ let $\eta_{a b} \in h\left(\Lambda_{a b}\right)$ be the image of $\eta$ under $\pi: \Lambda \rightarrow \Lambda_{a b}$. Note that $\eta_{a b}$ is just a morphism $A \rightarrow \Lambda_{a b}$. Given $g: A \rightarrow \Lambda_{a b}$, the preimage of $g$ under $\operatorname{Hom}\left(A, \Lambda_{a b} \oplus I\right) \rightarrow$ $\operatorname{Hom}\left(A, \Lambda_{a b}\right)$, is $\operatorname{Der}(A, I)$ (here the structure of A-module on I is given by $g$ ). Thus the map (2.3.7) defines, for any $\eta \in h(\Lambda)$, the action of $\operatorname{Der}(A, I)$ (with A-module structure on $I$ given by $\eta_{a b}$ ) on $h(p)^{-1} \eta \subset h\left(\Lambda^{\prime}\right)$. Properties (1) and (2) imply that this action makes $h(p)^{-1} \eta$ into a principal homogeneous space over $\operatorname{Der}(A, I)$. The same conclusion holds for $h^{R}$. But since $\xi_{*}^{\Lambda^{\prime}}$ takes, for any $\eta \in h(\Lambda)$,

$$
h(p)^{-1}(\eta) \rightarrow h^{\Lambda^{\prime}}(p)^{-1}\left(\xi_{*}^{\Lambda}(p)\right)
$$

and is a morphism of principal homogeneous spaces, it is a bijection. Theorem is proved. 


\section{$\S 3$. The affine NC-space and Feynman-Maslov operator calculus.}

(3.1) Free associative algebras.Let $V$ be a vector space and $\mathcal{A} s s(V)=\bigoplus_{d \geq 0} V^{\otimes d}$ be the tensor (free associative) algebra generated by $V$. Clearly, $\mathcal{A} s s(V)_{a b}=S(V)$ is the symmetric algebra of $V$. When $V=\mathbf{C}^{n}$ with basis $x_{1}, \ldots, x_{n}$, we identify $\mathcal{A s s}(V)$ with $\mathbf{C}\left\langle x_{1}, \ldots, x_{n}\right\rangle$ and $S(V)$ with $\mathbf{C}\left[x_{1}, \ldots, x_{n}\right]$.

(3.1.1) Definition. The affine $N C$-space $A_{N C}^{n}$ is the $N C$-scheme $\operatorname{Spf}\left(\mathbf{C}\left\langle x_{1}, \ldots, x_{n}\right\rangle_{\llbracket a b \rrbracket}\right)$.

Thus, as a ringed space, $A_{N C}^{n}$ is the ordinary affine space $A^{n}$ equipped with a certain sheaf $\mathcal{O}^{N C}$ of noncommutative algebras on the Zariski topology of $A^{n}$. The aim of this section is to describe $\mathcal{O}^{N C}$ explicitly by using the so-called calculus of ordered operators as developed by Feynman, Maslov and Karasev [Fe][Mas][KM]. The essence of this approach is to represent elements of $\mathbf{C}\left\langle x_{1}, \ldots, x_{n}\right\rangle$ by ordinary polynomials in $N \geq n$ variables. Let $[n]=\{1,2, \ldots, n\}$. Let $\tau:[N] \rightarrow[n]$ be any map and $f \in \mathbf{C}\left[y_{1}, \ldots, y_{N}\right]$ be a polynomial. Define a noncommutative polynomial

$$
\llbracket f(\stackrel{\tau}{x}) \rrbracket=\llbracket f\left(\stackrel{1}{x}_{\tau(1)}, \ldots, \stackrel{N}{x}{ }_{\tau(N)}\right) \rrbracket \in \mathbf{C}\left\langle x_{1}, \ldots, x_{N}\right\rangle
$$

by replacing each monomial $c \cdot y_{1}^{i_{1}} \ldots y_{N}^{i_{N}}$ in $f$ by $c \cdot x_{\tau(1)}^{i_{1}} x_{\tau(2)}^{i_{2}} \ldots x_{\tau(n)}^{i_{n}}$. For example, let $N=n=2$ and $f\left(y_{1}, y_{2}\right)=\left(y_{1}+y_{2}\right)^{2}$. Taking $\tau=\operatorname{Id}$ and $\sigma=(12)$ we get

$$
\llbracket f(\stackrel{\tau}{x}) \rrbracket=\left(\stackrel{1}{x}_{1}+\stackrel{2}{x}_{2}\right)^{2}=x_{1}^{2}+2 x_{1} x_{2}+x_{2}^{2}, \quad \llbracket f(\stackrel{\sigma}{x}) \rrbracket=\left(\stackrel{2}{x}_{1}+\stackrel{1}{x}_{2}\right)^{2}=x_{1}^{2}+2 x_{2} x_{1}+x_{2}^{2} .
$$

Thus the numbers over the variables indicate the order. Given $f \in \mathbf{C}\left[x_{1}, \ldots, x_{n}\right]$ we will use the default notation for the standard ordering $(\tau=\mathrm{Id})$ :

$$
\llbracket f(x) \rrbracket=\llbracket f\left(x_{1}, \ldots, x_{n}\right) \rrbracket=\llbracket f\left(\stackrel{1}{x}_{1}, \ldots, \stackrel{n}{x}_{n}\right) \rrbracket .
$$

(3.1.2) Proposition. $\mathbf{C}\left\langle x_{1}, \ldots, x_{n}\right\rangle$ can be identified with the space of formal finite sums

$$
\sum_{N, \tau} \llbracket f_{\tau}(\stackrel{\tau}{x}) \rrbracket, \quad f_{\tau} \in \mathbf{C}\left[y_{1}, \ldots, y_{N}\right]
$$

modulo the following cancellation rules:

(C1) If $f_{\tau} \in \mathbf{C}\left[y_{1}, \ldots, y_{N}\right]$ and $\tau(i)=\tau(i+1)$, then

$$
\llbracket f_{\tau}(\stackrel{\tau}{x}) \rrbracket=\llbracket\left(r_{i} f\right)\left(\stackrel{1}{x}_{\tau(1)}, \ldots, \stackrel{i}{x}_{\tau(i)}, \stackrel{i+1}{x}_{\tau(i+2)}, \ldots,{ }^{N-1} x_{\tau(N)}\right) \rrbracket,
$$

where $r_{i} f \in \mathbf{C}\left[y_{1}, \ldots, y_{N-1}\right]$ is defined by

$$
\left(r_{i} f\right)\left(y_{1}, \ldots, y_{N-1}\right)=f\left(y_{1}, \ldots, y_{i}, y_{i}, y_{i+1}, \ldots, y_{N-1}\right) .
$$


(C2) If $g \in \mathbf{C}\left[y_{1}, \ldots, y_{N-1}\right]$ and $s_{i} g \in \mathbf{C}\left[y_{1}, \ldots, y_{N}\right]$ is defined by

$$
\left(s_{i} g\right)\left(y_{1}, \ldots, y_{N}\right)=g\left(y_{1}, \ldots, \hat{y}_{i}, \ldots, y_{N}\right),
$$

then

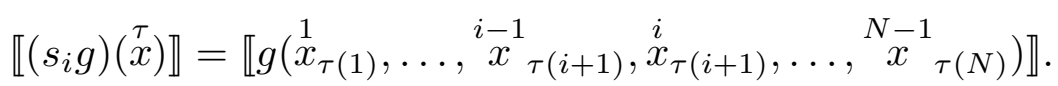

Proof: Given any $n$ associative algebras, $A_{1}, \ldots, A_{n}$, their free product $A_{1} * \cdots * A_{n}$ is spanned by formal products $a_{\tau(1)} * \cdots * a_{\tau(N)}$, where $N \in \mathbf{Z}_{+}, \tau:[N] \rightarrow[n]$, and $a_{\nu} \in A_{\nu}$. These products are $\mathbf{C}$-multilinear and are subject to the two cancellation rules:

$\left(\mathrm{C} 1^{\prime}\right) a_{i} * b_{i}=a_{i} b_{i}, a_{i} b_{i} \in A_{i}$.

$\left(\mathrm{C} 2^{\prime}\right) a_{i} * 1_{j} * a_{k}=a_{i} * a_{k}$, where $a_{i} \in A_{i}, a_{k} \in A_{k}$ and $1_{j} \in A_{j}$ is the unit.

So, $A_{1} * \cdots * A_{n}$ is a quotient of $\bigoplus_{N, \tau} A_{\tau(1)} \otimes \cdots \otimes A_{\tau(N)}$. When $A_{1}=\cdots=A_{n}=\mathbf{C}[x]$, we have

$$
A_{1} * \cdots * A_{n}=\mathbf{C}\left\langle x_{1}, \ldots, x_{n}\right\rangle, \quad A_{\tau(1)} \otimes \cdots \otimes A_{\tau(N)}=\mathbf{C}\left[y_{1}, \ldots, y_{N}\right]
$$

and $\left(\mathrm{C}^{\prime}\right),\left(\mathrm{C} 2^{\prime}\right)$ become $(\mathrm{C} 1)$ and $(\mathrm{C} 2)$.

(3.2) Free Lie algebras and the normal form in $\mathbf{C}\left\langle x_{1}, \ldots, x_{n}\right\rangle$. For a vector space $V$ let $\mathcal{L} i e(V)$ be the free Lie algebra generated by $V$. It is graded:

$$
\mathcal{L} i e(V)=\bigoplus_{d \geq 1} \mathcal{L} i e^{d}(V), \quad \mathcal{L} i e^{1}(V)=V .
$$

Every associative algebra, in particular, $\mathcal{A} s s(V)$, can be regarded as a Lie algebra via $[a, b]=a b-b a$. The following fact is classical.

(3.2.1) Theorem. (a) $\mathcal{L} i e(V)$ is isomorphic to the Lie subalgebra in $\mathcal{A} s s(V)$ generated by $V$.

(b) The embedding $\mathcal{L} i e(V) \subset \mathcal{A} s s(V)$ identifies $\mathcal{A} s s(V)$ with the universal enveloping algebra of $\mathcal{L} i e(V)$.

(3.2.2) Corollary. Let $\left\{\beta_{i}\right\}, i=1,2, \ldots$, be any $\mathbf{C}$-basis of $\mathcal{L} i e(V)$. Then the ordered monomials $\beta_{1}{ }^{m_{1}} \beta_{2}{ }^{m_{2}} \ldots$, where $m_{i} \in \mathbf{Z}_{+}$are 0 for almost all $i$, form a $\mathbf{C}$-basis in $\mathcal{A s s}(V)$.

We now take $V=\mathbf{C}^{n}$ with basis $x_{1}, \ldots, x_{n}$ and denote $\mathcal{L} i e(V)$ by $\mathcal{L}\left(x_{1}, \ldots, x_{n}\right)$ and $\mathcal{L} i e^{d}(V)$ by $\mathcal{L}^{d}\left(x_{1}, \ldots, x_{n}\right)$. Every element of $\mathcal{L}^{d}\left(x_{1}, \ldots, x_{n}\right)$ can be represented (not uniquely) as a linear combination of Lie monomials in $x_{1}, \ldots, x_{n}$. For every $d \geq 2$ choose an ordered basis $B_{d}$ in $\mathcal{L}^{d}\left(x_{1}, \ldots, x_{n}\right)$ (for example, one can take the Hall basis described in [MKS], $\S 5.6$, Ex. 10). Let $B=\coprod_{d \geq 2} B_{d}$ ordered lexicographically ( $B_{d}$ precedes $B_{d^{\prime}}$ if $d<d^{\prime}$ ). For $\beta \in B_{d}$ we write $\operatorname{ord}_{\mathrm{NC}}(\beta)=d-1$ (this is the total number of bracket pairs). Let us number the elements of $B$ according to their total order as $\beta_{1}, \beta_{2}, \ldots$. Denote by $\Lambda$ the set of all functions $\lambda: B \rightarrow \mathbf{Z}_{+}$with finite support. For $\lambda \in \Lambda$ we set

$$
M_{\lambda}(x)=\beta_{1}^{\lambda\left(\beta_{1}\right)} \beta_{2}^{\lambda\left(\beta_{2}\right)} \ldots \in \mathbf{C}\left\langle x_{1}, \ldots, x_{n}\right\rangle, \quad \operatorname{ord}(\lambda)=\sum_{\beta \in B} \lambda(\beta) \operatorname{ord}_{\mathrm{NC}}(\beta) \in \mathbf{Z} .
$$

The following is a reformulation of Corollary 3.2.2. 
(3.2.3) Proposition. Every element of $\mathbf{C}\left\langle x_{1}, \ldots, x_{n}\right\rangle$ can be uniquely written as a finite sum

$$
\sum_{\lambda \in \Lambda} \llbracket f_{\lambda}(x) \rrbracket M_{\lambda}(x)
$$

(3.3) Manipulations with ordered symbols. Here we recall several formulas due to Maslov and Karasev [Mas] [KM].

For a polynomial $f \in \mathbf{C}[\xi]$ its difference derivative $\partial f$ is defined by

$$
\partial f\left(\xi^{\prime}, \xi^{\prime \prime}\right)=\frac{\partial f}{\partial \xi}\left(\xi^{\prime}, \xi^{\prime \prime}\right)=\frac{f\left(\xi^{\prime}\right)-f\left(\xi^{\prime \prime}\right)}{\xi^{\prime}-\xi^{\prime \prime}} .
$$

The $m$-fold iterated difference derivative depends only on $m+1$ variables, reducing to

$$
\frac{\partial^{m} f}{\partial \xi^{m}}\left(\xi^{(0)}, \ldots, \xi^{(m)}\right)=\sum_{j=0}^{m} \frac{f\left(\xi^{(j)}\right)}{\prod_{i \neq j}\left(\xi^{(j)}-\xi^{(i)}\right)}
$$

Accordingly, for a polynomial $f\left(\xi_{1}, \ldots, \xi_{n}\right)$ in $n$ variables its partial difference derivative

$$
\frac{\partial^{m_{1}+\cdots+m_{n}} f}{\partial \xi_{1}^{m_{1}} \cdots \partial \xi_{n}^{m_{n}}}
$$

depends on $\left(m_{1}+1\right)+\cdots+\left(m_{n}+1\right)$ variables $\xi_{i}^{(j)}, 0 \leq j \leq m_{i}$. Now the main formula of Maslov ([Mas], Ch. 0, Th. 4.3) is:

(3.3.1) Change of order formula I. For any $g \in \mathbf{C}\left[y_{1}, y_{2}\right]$ we have an equality in $\mathbf{C}\langle a, b\rangle$ :

$$
\llbracket g(\stackrel{2}{a}, b) \rrbracket-\llbracket g(\stackrel{1}{a}, \stackrel{2}{b}) \rrbracket=\llbracket\left[a^{3}, b\right] \frac{\delta^{2} g}{\delta y_{1} \delta y_{2}}(\stackrel{2}{a}, \stackrel{4}{a}, \vec{b}, \vec{b}) \rrbracket .
$$

By iterating this, one gets a more precise formula involving the usual partial derivatives ([KM] App. 1, Th. 1.9):

(3.3.2) Change of order formula II. In the above situation we have

$$
\llbracket g(\stackrel{2}{a}, \stackrel{1}{b}) \rrbracket=\sum_{m, l \geq 0} \llbracket \frac{\partial^{l+m} g}{\partial y_{1}^{l} \partial y_{2}^{m}}(\stackrel{1}{a}, \stackrel{3}{a}) \stackrel{2}{K_{l, m}} \rrbracket,
$$

where

$$
K_{l, m}=\frac{1}{l ! m !} \llbracket(\stackrel{1}{a}-\stackrel{3}{a})^{l}(\stackrel{2}{a}-b)^{m} \rrbracket
$$

satisfies $\operatorname{ord}_{\mathrm{NC}}\left(K_{l, m}\right) \geq \max (l, m)$.

To write down $K_{l, m}$ one should first think of the $\stackrel{i}{a}$ as different variables, expanding the powers by the binomial formula, and then make out of every commutative monomial in $\stackrel{1}{a}, \stackrel{2}{a}, \stackrel{3}{a}, b$ a noncommutative monomial in $a, b$, as indicated by the superscripts.

One has similar formulas for interchanging the order of any two consecutive variables in a many-variable symbol $g\left(y_{1}, \ldots, y_{N}\right)$, see e.g. [Mas]. For the next formula, see $[\mathrm{KM}]$, App. 1, n. (1.3). 
(3.3.3) Commutation formula. For any $f \in \mathbf{C}\left[y_{1}, \ldots, y_{n}\right]$ we have the identity in $\mathbf{C}\left\langle x_{1}, \ldots, x_{n}, a\right\rangle$ :

$$
\begin{aligned}
a \cdot \llbracket f\left(x_{1}, \ldots, x_{n}\right) \rrbracket= & \sum_{i_{1}, \ldots, i_{n}=0}^{\infty} \frac{1}{i_{1} ! \ldots i_{n} !} \llbracket \frac{\partial^{i_{1}+\cdots+i_{n}} f}{\partial y_{1}^{i_{1}} \ldots \partial y_{n}^{i_{n}}}\left(x_{1}, \ldots, x_{n}\right) \rrbracket . \\
& \cdot \operatorname{ad}\left(x_{n}\right)^{i_{n}} \ldots \operatorname{ad}\left(x_{1}\right)^{i_{1}}(a) .
\end{aligned}
$$

(3.3.4) Taylor formula. For any $f(y) \in \mathbf{C}[y]$ we have the equality in $\mathbf{C}\langle a, b\rangle$ :

$$
f(a+b)=\sum_{k \geq 0} \llbracket f^{(k)}(\stackrel{1}{a}+\stackrel{3}{b}) X_{k}^{2} \rrbracket,
$$

where

$$
X_{k}=\frac{1}{k !} \llbracket\left(\frac{2}{a+b}-\stackrel{1}{a}-\stackrel{3}{b}\right)^{k} \rrbracket \in \mathbf{C}\langle a, b\rangle
$$

satisfies $\operatorname{ord}_{\mathrm{NC}}\left(X_{k}\right) \geq[(k+1) / 2]$.

(3.4) Multiplication of elements in the normal form. The formulas (3.3.1-3) can be used as an algorithm for bringing the product

$$
\left(\sum_{\lambda \in \Lambda} \llbracket f_{\lambda}(x) \rrbracket M_{\lambda}(x)\right)\left(\sum_{\mu \in \Lambda} \llbracket g_{\mu}(x) \rrbracket M_{\mu}(x)\right) \in \mathbf{C}\left\langle x_{1}, \ldots, x_{n}\right\rangle
$$

of two elements given in the normal form, back into the normal form, i.e., expressing it as

$$
\sum_{\nu \in \Lambda} \llbracket h_{\nu}(x) \rrbracket M_{\nu}(x) .
$$

More precisely, we have the following fact.

(3.4.3) Proposition. For every $\lambda, \mu, \nu \in \Lambda$ there is unique bilinear differential operator with polynomial coefficients

$$
C_{\lambda \mu}^{\nu}=C_{\lambda \mu}^{\nu}(f, g): \mathbf{C}\left[x_{1}, \ldots, x_{n}\right] \underset{\mathbf{C}}{\otimes} \mathbf{C}\left[x_{1}, \ldots, x_{n}\right] \rightarrow \mathbf{C}\left[x_{1}, \ldots, x_{n}\right]
$$

with the following properties:

(a) In the normal form (3.4.2) for $\llbracket f(x) \rrbracket M_{\lambda}(x) \cdot \llbracket g(x) \rrbracket \cdot M_{\mu}(x)$ we have $h_{\nu}=C_{\lambda \mu}^{\nu}(f, g)$.

(b) $C_{\lambda \mu}^{\nu}=0$ if $\operatorname{ord}(\nu)<\operatorname{ord}(\lambda)+\operatorname{ord}(\mu)$ as well as if $\operatorname{ord}(\nu)=\operatorname{ord}(\lambda)+\operatorname{ord}(\mu)$ and $\nu \neq \lambda+\mu$, while $C_{\lambda \mu}^{\lambda+\mu}$ is the operator of multiplication of polynomials.

(c) The $C_{\lambda \mu}^{\nu}$ satisfy the associativity constraint: for any $\lambda_{1}, \lambda_{2}, \lambda_{3}, \nu \in \Lambda$ the trilinear differential operators $\sum_{\mu_{1}} C_{\mu_{1}, \lambda_{3}}^{\nu} \circ\left(C_{\lambda_{1}, \lambda_{2}}^{\mu_{1}} \otimes 1\right)$ and $\sum_{\mu_{2}} C_{\lambda_{1}, \mu_{2}}^{\nu} \circ\left(1 \otimes C_{\lambda_{2}, \lambda_{3}}^{\mu_{2}}\right)$ coincide.

Proof: We first establish the existence of the $C_{\lambda \mu}^{\nu}$ satisfying (a). In order to bring $\llbracket f(x) \rrbracket M_{\lambda}(x) \llbracket g(x) \rrbracket \cdot M_{\mu}(x)$ into the normal form, we need only to do this for the intermediate product, which is $M_{\lambda}(x) \llbracket g(x) \rrbracket$ for $\lambda \neq 0$ and $\llbracket f(x) \rrbracket \cdot \llbracket g(x) \rrbracket$ if $\lambda=0$ (after 
this, the transforming of $M_{\nu}(x) M_{\mu}(x)$ to a linear combination of the $M_{\sigma}(x)$ is a purely Lie-algebraic procedure not affecting the coefficients of the form $\llbracket h(x) \rrbracket)$.

Now, if $\lambda \neq 0$, we apply (3.3.3) to $a=M_{\lambda}(x)$ to express $M_{\lambda}(x) \llbracket g(x) \rrbracket$ as a sum of terms in which ordered symbols are on the left and commutators are on the right and then use the Jacobi identity to express each commutator via basic Lie monomials from $B$.

If $\lambda=0$, we have

$$
\llbracket f(x) \rrbracket \llbracket g(x) \rrbracket=\llbracket(f \otimes g)\left(\stackrel{1}{x}_{1}, \ldots, \stackrel{n}{x}_{n}, \stackrel{n+1}{x}_{1}, \ldots, \stackrel{2 n}{x}_{n}\right) \rrbracket,
$$

where

$$
(f \otimes g)\left(y_{1}, \ldots, y_{2 n}\right)=f\left(y_{1}, \ldots, y_{n}\right) g\left(y_{n+1}, \ldots, y_{2 n}\right)
$$

By using (3.3.2), we move the second copies of the $x_{i}$ under $f \otimes g$ to the left with the aim being to bring the second copy of $x_{i}$ in adjacency with the first and use the cancellation rule (C1) of (3.1.2). Each step of this process creates several new terms involving commutators which, in their turn are not in the normal form, so we apply (3.3.2) to them and so on.

This proves the existence of $C_{\lambda \mu}^{\nu}$. The uniqueness follows since two bilinear differential operators with polynomial coefficients whose values on every pair of polynomials coincide, should be equal as formal expressions. Thus (a) is proved. Part (b) is clear from the nature of the formulas (3.3.2-3). Finally, (c) follows from the associativity of $\mathbf{C}\left\langle x_{1}, \ldots, x_{n}\right\rangle$ and the fact that a trilinear differential operator with polynomial coefficients is uniquely defined by its values on all triples of polynomials.

(3.4.4) Example. Working modulo $F^{2}$, i.e., setting

$$
\left[x_{i}, x_{j}\right] \varphi\left[x_{k} x_{l}\right]=\left[x_{i}\left[x_{j}, x_{k}\right]\right]=0
$$

we find

$$
\llbracket f(x) \rrbracket \cdot \llbracket g(x) \rrbracket=\llbracket(f g)(x) \rrbracket+\sum_{j>i} \llbracket\left(\frac{\partial f}{\partial x_{j}} \cdot \frac{\partial g}{\partial x_{i}}\right)(x) \rrbracket \cdot\left[x_{i}, x_{j}\right]
$$

which is equivalent to the formula of Example 1.3.9.

(3.4.5) Corollary. The $N C$-filtration on $\mathbf{C}\left\langle x_{1}, \ldots, x_{n}\right\rangle$ is given by $F^{d} \mathbf{C}\left\langle x_{1}, \ldots, x_{n}\right\rangle=$ $\left\{\sum_{\operatorname{ord}(\lambda) \geq d} \llbracket f_{\lambda}(x) \rrbracket M_{\lambda}(x)\right\}$.

Proof: Denote the LHS of the proposed equality by $F^{d}$ and the RHS by $J^{d}$. By definition of $M_{\lambda}(x)$, we have $J^{d} \subset F^{d}$. On the other hand, (3.4.3)(b) implies

$$
J^{d} \cdot J^{d^{\prime}} \subset J^{d+d^{\prime}}, \quad\left[J^{d}, J^{d^{\prime}}\right] \subset J^{d+d^{\prime}+1},
$$

which entails $F^{d} \subset J^{d}$.

We now reformulate this in a more invariant form. Let $V$ be a finite-dimentinal vector space. Consider the graded vector space $\mathcal{L} i e_{+}(V)=\oplus_{d \geq 2} \mathcal{L} i e^{d}(V)$ with the grading given 
by $\operatorname{deg}\left(\mathcal{L} i e^{d}(V)\right)=d-1$. Introduce in the symmetric algebra $S\left(\mathcal{L} i e_{+}(V)\right)$ the induced grading and set

$$
Q^{d}(V)=S\left(\mathcal{L} i e_{+}(V)\right)^{d}
$$

to be the $d$ th homogeneous part. Clearly $Q^{d}$ is a polynomial functor on the category of vector spaces [Mac].

(3.4.6) Proposition. We have a natural identification

$$
Q^{d}(V) \simeq F^{d} \mathcal{A} s s(V) \cap V^{\otimes d}
$$

If $V=\bigoplus_{i=1}^{n} \mathbf{C} \cdot x_{i}$, so that $\mathcal{A s s}(V)=\mathbf{C}\left\langle x_{1}, \ldots, x_{n}\right\rangle$, then the $M_{\lambda}(x)$, $\operatorname{ord}(\lambda)=d$, form a basis of $Q^{d}(V)$.

Proof: By construction of $F^{d}$, we have $F^{d+1} \mathcal{A} s s(V) \cap V^{\otimes d}=0$, thus

$$
F^{d} \mathcal{A} s s(V) \cap V^{\otimes d} \simeq \operatorname{gr}_{F}^{d} \mathcal{A} s s(V) \cap V^{\otimes d}
$$

is identified with a part of the Poisson algebra $\operatorname{gr}_{F}^{\bullet} \mathcal{A} s s(V)$. The (commutative) multiplication and the Poisson bracket in this algebra define a map $\varphi: Q^{d}(V) \rightarrow \operatorname{gr}_{F}^{d} \mathcal{A} s s(V)$ whose image is contained in the image of $F^{d} \mathcal{A} s s(V) \cap V^{\otimes d}$, so we get

$$
\psi: Q^{d}(V) \rightarrow F^{d} \mathcal{A} s s(V) \cap V^{\otimes d}
$$

To see that $\psi$ is an isomorphism, choose a basis $x_{i}, \ldots, x_{n}$ in $V$. Then the $M_{\lambda}(x)$, ord $(\lambda)=$ $d$ can be seen as the images, under $\psi$, of the elements of the basis of $Q^{d}(V)=S\left(\mathcal{L} i e_{+}(V)\right)^{d}$ formed by products of the basic Lie monomials in the $x_{i}$. On the other hand, an element $\sum_{\operatorname{ord}(\lambda) \geq d} \llbracket f_{\lambda}(s) \rrbracket M_{\lambda}(x)$ lies in $V^{\otimes d}$ if and only if $f_{\lambda}=0$ for $\operatorname{ord}(\lambda)>d$ and $f_{\lambda} \in \mathbf{C}$ for $\operatorname{ord}(\lambda)=d$. This proves that the $M_{\lambda}(x)$, ord $(\lambda)=d$, form a C-basis in $F^{d} \mathcal{A} s s(V) \cap V^{\otimes d}$ and that $\psi$ is an isomorphism.

(3.4.7) Proposition. We have a natural identification of $G L(V)$-equivariant $S(V)$-modules:

$$
\operatorname{gr}_{F}^{d} \mathcal{A} s s(V) \simeq S(V) \underset{\mathbf{C}}{\otimes} Q^{d}(V)
$$

Proof: The identification of (3.4.6) gives a morphism of $S(V)$-modules

$$
S(V) \otimes Q^{d}(V) \rightarrow \operatorname{gr}_{F}^{d} \mathcal{A} s s(V)
$$

To see that it is an isomorphism, we choose a basis in $V$ and use (3.4.5).

Another corollary of (3.4.5) is as follows. 
(3.4.8) Proposition. The algebra $\mathbf{C}\left\langle x_{i}, \ldots, x_{n}\right\rangle_{\llbracket a b \rrbracket}$ can be identified with the set of possibly infinite formal sums $\sum_{\lambda \in \Lambda} \llbracket f_{\lambda}(x) \rrbracket M_{\lambda}(x)$ and multiplication given by the operators $C_{\lambda \mu}^{\nu}$.

(3.5) Explicit description of $\mathcal{O}_{A^{n}}^{\mathrm{NC}}$. Let $\mathcal{D}_{n}$ be the Weyl algebra of differential operators in $\mathbf{C}\left[x_{1}, \ldots, x_{n}\right]$. By a $\mathcal{D}_{n}$-algebra we will mean a left $\mathcal{D}_{n}$-module $A$ equipped with a commutative algebra structure such that the action of the $\partial / \partial x_{i}$ is by algebra derivations. For example, any localization $\mathbf{C}\left[x_{i}, \ldots, x_{n}\right]\left[S^{-1}\right]$ is a $\mathcal{D}_{n}$-algebra. Note that multilinear differential operators with polynomial coefficients can be evaluated on elements of $A$.

(3.5.1) Definition. Let $A$ be a $\mathcal{D}_{n}$-algebra. The algebra $\mathbf{C}\left\langle x_{i}, \ldots, x_{n}\right\rangle_{\llbracket a b \rrbracket} \otimes_{\mathbf{C}\left[x_{1} \ldots x_{n}\right]} A$ is defined as the space of possibly infinite formal expressions $\sum_{\lambda \in \Lambda} \llbracket f_{\lambda} \rrbracket M_{\lambda}(x), f_{\lambda} \in A$ and the multiplication given by

$$
\llbracket f_{\lambda} \rrbracket M_{\lambda}(x) \cdot \llbracket f_{\mu} \rrbracket M_{\mu}(x)=\sum_{\nu} \llbracket C_{\lambda \mu}^{\nu}\left(f_{\lambda}, f_{\mu}\right) \rrbracket M_{\mu}(x),
$$

where $C_{\lambda \mu}^{\nu}$ was introduced in (3.4.3).

(3.5.2) Proposition. The above multiplication is well defined and makes

$$
\mathbf{C}\left\langle x_{1}, \ldots, x_{n}\right\rangle_{\llbracket a b \rrbracket} \underset{\mathbf{C}\left[x_{1} \ldots x_{n}\right]}{\otimes} A
$$

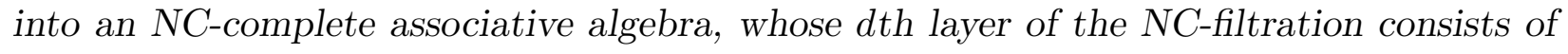
elements of the form $\sum_{\operatorname{ord}(\lambda) \geq d} \llbracket f_{\lambda} \rrbracket M_{\lambda}(x)$.

Proof: Well-definedness: we need only to show that the product of two infinite sums $\left(\sum_{\lambda} \llbracket f_{\lambda} \rrbracket M_{\lambda}(x)\right)\left(\sum_{\mu} \llbracket g_{\mu} \rrbracket M_{\mu}(x)\right)$ makes sense, i.e. the number of pairs $\lambda, \mu$ such that $C_{\lambda \mu}^{\nu} \neq 0$, is finite for every $\nu$. But this follows from (3.4.3)(b). Associativity follows from $(3.4 .3)(\mathrm{c})$. The statement about the NC-filtration is proved in the same way as (3.4.5). The NC-completeness follows from that.

(3.5.3) Theorem. Let $U \subset A^{n}$ be a Zariski open set, $\mathcal{O}(U)$ be the algebra of rational functions regular in $\mathrm{U}$ and

$$
\mathcal{A}(U)=\mathbf{C}\left\langle x_{1}, \ldots, x_{n}\right\rangle_{\llbracket a b \rrbracket} \underset{\mathbf{C}\left[x_{1}, \ldots, x_{n}\right]}{\otimes} \mathcal{O}(\mathrm{U}) .
$$

Then $\Gamma\left(U, \mathcal{O}_{A^{n}}^{\mathrm{NC}}\right)$ is naturally identified with $\mathcal{A}(U)$.

Proof: The $\mathcal{A}(U)$ obviously form a sheaf $\mathcal{A}$ of NC-complete algebras on $A^{n}$. For $d \geq 0$ let $\mathcal{A}^{(d)}=\mathcal{A} / F^{d+1}$ be the sheaf formed by expressions $\sum_{\operatorname{ord}(\lambda) \leq d} \llbracket f_{\lambda} \rrbracket M_{\lambda}(x)$ (with all the $\llbracket h_{\nu} \rrbracket M_{\nu}(x), \operatorname{ord}(\nu)>d$ in the product of two such expression being discarded). Denote by $q_{d}: \mathcal{A}^{(d)} \rightarrow \mathcal{A}^{(d-1)}$ the natural projection. Let also $R^{(d)}=\mathbf{C}\left\langle x_{1}, \ldots, x_{n}\right\rangle / F^{d+1}$ and $\mathcal{O}^{(d)}$ be the sheaf of algebras on $A^{n}$ corresponding to $\operatorname{Spec}\left(R^{(d)}\right)=\left(A^{n}, \mathcal{O}^{(d)}\right)$. Denote 
$p_{d}: \mathcal{O}^{(d)} \rightarrow \mathcal{O}^{(d-1)}$ the natural projection. To prove our theorem, it is enough to construct, for each $d$, an isomorphism $\varphi_{d}: \mathcal{O}^{(d)} \rightarrow \mathcal{A}^{(d)}$ of sheaves of algebras, in a way compatible with the $p_{d}, q_{d}$.

Construction of $\varphi_{c}$. Let $U=\{f \neq 0\}$ be a principal open subset in $A^{n}$, so $\mathcal{O}(U)=$ $\mathbf{C}\left[x_{1}, \ldots, x_{n}\right]\left[f^{-1}\right]$. Let $\llbracket f \rrbracket \in \mathbf{C}\left\langle x_{1}, \ldots, x_{n}\right\rangle$ be the ordered lifting of $f$. Then $\llbracket f \rrbracket_{a b}=f$. Note that $\Gamma\left(U, \mathcal{O}^{(d)}\right)=R^{(d)}\left[\llbracket f \rrbracket^{-1}\right]$ (any $g$ with $g_{a b}=f$ will become invertible after $\llbracket f \rrbracket$ is inverted). On the other hand, we have an NC-nilpotent algebra $\mathcal{A}^{(d)}(U)$ with $\mathcal{A}^{(d)}(U)_{a b}=\mathcal{O}(U)$, containing $R^{(d)}$. In this algebra $\llbracket f \rrbracket$ is invertible (because $f=\llbracket f \rrbracket_{a b}$ is invertible in $\mathcal{O}(U)$, see Proposition 2.1.1). Thus, by the universal property of the ring of fractions, we have a homomorphism $\varphi_{d, U}: \mathcal{O}^{(d)}(U) \rightarrow \mathcal{A}^{(d)}(U)$. From these we construct a morphism of sheaves $\varphi_{d}: \mathcal{O}^{(d)} \rightarrow \mathcal{A}^{(d)}$ in a standard way. It is clear that $q_{d} \varphi_{d}=\varphi_{d-1} p_{d}$.

$\varphi_{d}$ is an isomorphism. Since $\varphi_{d}$ (as any homomorphism of sheaves of algebras) takes $F^{i} \mathcal{O}^{(d)} \rightarrow F^{i} \mathcal{A}^{(d)}$, it suffices to prove that $\bar{\varphi}_{d}: \operatorname{gr}_{F}^{\bullet} \mathcal{O}^{(d)} \rightarrow \operatorname{gr}_{F}^{\bullet} \mathcal{A}^{(d)}$ is an isomorphism. As before, it is enough to do this over a principal open subset $U=\{f \neq 0\}$. In this case, by Theorem 2.1.6,

$$
\operatorname{gr}_{F}^{\bullet} \mathcal{O}^{(d)}(U)=\operatorname{gr}_{F}^{\bullet}\left(R^{(d)}\left[\llbracket f \rrbracket^{-1}\right]\right)=\operatorname{gr}_{F}^{\bullet}\left(R^{(d)}\right)\left[f^{-1}\right]
$$

But $\operatorname{gr}_{F}^{i} R^{(d)}$ is equal to 0 for $i>d$ and to $\operatorname{gr}_{F}^{i} \mathbf{C}\left\langle x_{1}, \ldots, x_{n}\right\rangle$ for $i \leq d$. The latter is, by (3.4.6), a free $\mathbf{C}\left[x_{1}, \ldots, x_{n}\right]$-module with basis $M_{\lambda}(x)$, ord $(\lambda)=i$. Therefore $\operatorname{gr}_{F}^{\bullet} \mathcal{O}^{(d)}(U)$ is a free $\mathcal{O}(U)$-module with basis $M_{\lambda}(x) \operatorname{ord}(\lambda) \leq d$. From Proposition 3.5.1, we see that $\operatorname{gr}_{F}^{\bullet} \mathcal{A}^{(d)}(U)$ has the same form. Moreover, the morphism $\bar{\varphi}_{d}$ is the identity. Theorem is proved.

(3.5.4) Examples. (a) Let $n=2$, and $U \subset A^{2}$ be given by $x_{1} \neq 0$. We have the (noncommuting) elements $x_{i}=\llbracket x_{i} \rrbracket \in \mathcal{O}^{\mathrm{NC}}(U)$ with $x_{1}$ invertible. The two ordered quotients of $x_{2}$ by $x_{1}$ are written explicitly as follows:

$$
\begin{gathered}
x_{1}^{-1} x_{2}=\llbracket x_{2} / x_{1} \rrbracket \\
x_{2} x_{1}^{-1}=\llbracket x_{2} / x_{1} \rrbracket+\llbracket 1 / x_{1}^{2} \rrbracket\left[x_{1}, x_{2}\right]+\llbracket 1 / x_{1}^{3} \rrbracket\left[x_{1},\left[x_{1}, x_{2}\right]\right]+\cdots
\end{gathered}
$$

See (2.1.7.2) and (3.3.3).

(b) Let $n=2$ and $U \subset A^{2}$ given by $x_{1}+x_{2} \neq 0$. Identifying, as before, $x_{i}$ with $\llbracket x_{i} \rrbracket \in \mathcal{O}(U)$, we find that $x_{1}+x_{2}$ is invertible in $\mathcal{O}(U)$. The inverse can be written explicitly by applying the Taylor formula (3.3.4) to $f(z)=1 / z$, getting

$$
\left(x_{1}+x_{2}\right)^{-1}=\sum_{k=0}^{\infty}\left[\left[\frac{(-1)^{k} k !}{\frac{3}{x_{1}+x_{2}}} \cdot \stackrel{2}{X}_{k}\right]\right]
$$

Then we should bring each summand to the normal form by applying (3.3.2) to symbols like

$$
g\left(y_{1}, y_{2}, y_{3}\right)=\frac{y_{2}}{\left(y_{1}+y_{3}\right)^{k}}
$$


(c) Let $n=m^{2}$, so $A^{n}=\operatorname{Mat}_{m}(\mathbf{C})$ is the space of matrices. The matrix elements $x_{i j}$ are the coordinates in $A^{n}$. Let $\mathrm{U}=G L_{m}(\mathbf{C}) \subset A^{n}$ be given by $\operatorname{det}\left\|x_{i j}\right\| \neq 0$. Let us now identify the $x_{i j}$ with the noncommuting elements $\llbracket x_{i j} \rrbracket \in \mathcal{O}(U)$, By (2.1.1), the tautological matrix $\mathbf{M}=\left\|x_{i j}\right\| \in \operatorname{Mat}_{m}(\mathcal{O}(U))$ is invertible. So the entries $C_{i j}$ of $\mathbf{M}^{-1}$ are some series $\sum \llbracket f_{\lambda}(x) \rrbracket M_{\lambda}(x), f_{\lambda} \in \mathcal{O}\left(G L_{m}(\mathbf{C})\right)$.

(3.5.5) Theorem. The algebra $\mathbf{C}\left\langle\left\langle x_{i}, \ldots, x_{n}\right\rangle\right\rangle$ is naturally identified with

$$
\mathbf{C}\left\langle x_{i}, \ldots, x_{n}\right\rangle_{\llbracket a b \rrbracket} \otimes_{\mathbf{C}\left[x_{1} \ldots x_{n}\right]} \mathbf{C}\left[\left[x_{1} \ldots x_{n}\right]\right]
$$

Proof. Any formal sum $\sum_{\lambda \in \Lambda} \llbracket f_{\lambda}(x) \rrbracket M_{\lambda}(x)$ with $f_{\lambda}(x) \in \mathbf{C}\left[\left[x_{i}, \ldots, x_{n}\right]\right]$, can be regarded as a noncommutative power series in the $x_{i}$, so we have an embedding from one algebra to another. This embedding induces an isomorphism on $\operatorname{gr}_{F}^{\bullet}$. Both algebras being NC-complete, our embedding is an isomorphism. 


\section{$\S 4$. Detailed study of algebraic NC-manifolds.}

(4.1) Poisson envelopes. Let $\mathcal{P}$ ois be the category of Poisson algebras (1.1) and $c$ : $\mathcal{P}$ ois $\rightarrow$ Com the functor forgetting the Poisson bracket.

(4.1.1) Proposition. The functor $c$ has a left adjoint $P$ called the Poisson envelope.

Proof: Let $A$ be a commutative algebra and $\mathcal{P}$ ois $(A)$ be the free Poisson algebra generated by $A$ as a vector space. Let $\cdot,\{$,$\} be the operations in \mathcal{P}$ ois $(A)$. Define $P(A)$ as the quotient of $\mathcal{P}$ ois $(A)$ by the Poisson ideal generated by $a \cdot b-a b, a, b \in A$. Here $a b \in A$ is the product of $a$ and $b$ in $A$. The adjointness of $P$ and $c$ is obvious from the construction. (4.1.2) Example. If $A=\mathbf{C}\left[x_{1}, \ldots, x_{n}\right]$ is the free commutative algebra, then $P(A)=$ $\mathcal{P}$ ois $\left(x_{1}, \ldots, x_{n}\right)$ is the free Poisson algebra on $x_{1}, \ldots, x_{n}$. By (3.4.5-6) this algebra is canonically identified with $\operatorname{gr}_{F}^{\bullet} \mathbf{C}\left\langle x_{1}, \ldots, x_{n}\right\rangle$.

Note that by construction $P(A)=\bigoplus_{d \geq 0} P^{d}(A)$ is a graded (1.1.3) Poisson algebra, with $P^{d}(A)$ spanned by the images of formal expressions from $\operatorname{Pois}(A)$ containing $d$ instances of Poisson brackets. In particular $P^{0}(A)=A$ and each $P^{d}(A)$ is an $A$-module.

In (3.4) we defined a polynomial functor $Q^{d}$ on the category of vector spaces. Let $Q_{A}^{d}$ be the natural extension of $Q^{d}$ to the category of projective $A$-modules. For such a module $M$ the fiber of $Q_{A}^{d}(M)$ at a $\mathbf{C}$-point $x \in \operatorname{Spec}(A)$ is the value of $Q^{d}$ on the fiber of $M$ at $x$.

(4.1.3) Theorem. Let $A$ be a smooth, finitely generated, commutative algebra. Then $P^{d}(A) \simeq Q_{A}^{d}\left(\Omega_{A}^{1}\right)$ as an $A$-module.

Proof: We first construct an $A$-linear map $\varphi: P^{d}(A) \rightarrow Q_{A}^{d}\left(\Omega_{A}^{1}\right)$. The space $P^{d}(A)$ is spanned by products of the form $\pi=f_{0} h_{1} \cdots h_{m}$, where $f_{0} \in A$ and each $h_{v}$ is a bracket monomial

$$
h_{v}=\left\{f_{v, 0},\left\{f_{v, 1}, \ldots,\left\{f_{v, l_{v}-1}, f_{v, l_{v}}\right\} \ldots\right\}, \quad f_{v, j} \in A, \quad \sum_{v=1}^{m} l_{v}=d .\right.
$$

These products are considered modulo the Jacobi and Leibniz identities. For $\pi$ as above set

$$
\varphi(\pi)=f_{0} \cdot \prod_{v=1}^{m}\left[d f_{v, 0},\left[d f_{v, 1}, \ldots,\left[d f_{v, l_{v}-1}, d f_{v, l_{v}}\right] \ldots\right] \in Q_{A}^{d}\left(\Omega_{A}^{1}\right) .\right.
$$

Because $d: A \rightarrow \Omega_{A}^{1}$ satisfies the Leibniz rule, this is compatible with the Leibniz identity for the Poisson brackets. Because [, ] satisfies the Jacobi identity, $\varphi$ is compatible with the Jacobi identity for Poisson brackets. So $\varphi$ is a well defined $A$-module homomorphism.

We now prove that $\varphi$ is an isomorphism. Both the Poisson envelope and localization being direct limit-type constructions, we have

$$
P^{d}\left(A\left[S^{-1}\right]\right)=P^{d}(A)\left[S^{-1}\right] .
$$


Thus it is enough to prove that $\varphi$ is an isomorphism in the case when $A$ is a local ring, obtained by localizing a finitely generated smooth algebra at a $\mathbf{C}$-point. In this case we represent

$$
A=\mathbf{C}\left[x_{1}, \ldots, x_{n}\right]^{\sim} /\left(h_{1}, \ldots, h_{m}\right)
$$

where the superscript " $\sim$ " means localization at 0 and $h_{1}, \ldots, h_{m} \in \mathbf{C}\left[x_{1}, \ldots, x_{n}\right]$ are such that $d_{0} h_{i}$ are linearly independent. Then, denoting by $\left\{\left(h_{1}, \ldots, h_{m}\right)\right\} \subset \mathcal{P}$ ois $\left(x_{1}, \ldots, x_{n}\right)$ the Poisson ideal generated by the $x_{i}$, we have, in virtue of (4.1.4):

$$
P^{d}(A) \cong\left(\mathcal{P o i s}\left(x_{1}, \ldots, x_{n}\right) /\left\{\left(h_{1}, \ldots, h_{m}\right)\right\}\right)^{d} \underset{\mathbf{C}\left[x_{1} \ldots x_{n}\right]}{\otimes} \mathbf{C}\left[x_{1}, \ldots, x_{n}\right]^{\sim}
$$

Now, since

$$
\begin{gathered}
\operatorname{Pois}^{d}\left(x_{1}, \ldots, x_{n}\right) \simeq \mathbf{C}\left[x_{1}, \ldots, x_{n}\right] \underset{\mathbf{C}}{\otimes} Q^{d}\left(\oplus \mathbf{C} \cdot x_{i}\right) \simeq \\
\simeq Q_{\mathbf{C}\left[x_{1}, \ldots, x_{n}\right]}^{d}\left(\Omega_{\mathbf{C}\left[x_{1}, \ldots, x_{n}\right]}^{1}\right)
\end{gathered}
$$

and

$$
\Omega_{A}^{1} \simeq \Omega_{\mathbf{C}\left[x_{1}, \ldots, x_{n}\right] \sim}^{1} /\left(h_{i}, d h_{i}\right)
$$

we find that the factorization of $\mathcal{P}_{\text {ois }}{ }^{d}\left(x_{1}, \ldots, x_{n}\right)$ given by $\left\{\left(h_{1}, \ldots, h_{m}\right)\right\}$ exactly corresponds, via $\varphi$, to the factorization of $Q_{\mathbf{C}\left[x_{1}, \ldots, x_{n}\right]}^{d}\left(\Omega_{\mathbf{C}\left[x_{1}, \ldots, x_{n}\right]}^{1}\right)$ induced by (4.1.7). So $\varphi$ is an isomorphism and the theorem is proved.

Let now $M$ be a smooth algebraic variety. Because of (4.1.4), we have a sheaf $P\left(\mathcal{O}_{M}\right)$ of graded Poisson algebras on $M$ with $P^{0}\left(\mathcal{O}_{M}\right)=\mathcal{O}_{M}$ and $P^{d}\left(\mathcal{O}_{M}\right) \simeq Q^{d}\left(\Omega_{M}^{1}\right)$ as an $\mathcal{O}_{M}$-module. The definition of $Q^{d}$ implies:

(4.1.8) Proposition. The spectrum of $P\left(\mathcal{O}_{M}\right)$ is the (infinite-dimensional) manifold $\prod_{d \geq 2}{ }_{M} \mathcal{L} i e^{d}\left(T_{M}\right)$, i.e. the completion of the degree $\geq 2$ part of the free $\mathcal{O}_{M}$-Lie algebra generated by the tangent bundle $T_{M}$.

(4.1.9) Example. A Poisson structure $\xi$ on $M$ itself gives a section $M \rightarrow \operatorname{Spec}\left(P\left(\mathcal{O}_{M}\right)\right)$. Its composition with the projection to $\mathcal{L} i e^{2}\left(T_{M}\right)=\bigwedge^{2} T_{M}$ is the bivector corresponding to $\xi$ in a standard way.

\section{(4.2) Structure of $\operatorname{gr}_{F}^{\bullet}$ for $d$-smooth algebras.}

(4.2.1) Theorem. Let $R$ be a $d$-smooth algebra, $A=R_{a b}$. Then the Poisson algebra $\operatorname{gr}_{F}^{\bullet}(R)$ is canonically isomorphic to $P(A) / P^{\geq d+1}(A)$. In particular, $\operatorname{gr}_{F}^{i}(R) \simeq Q_{A}^{i}\left(\Omega_{A}^{1}\right)$ for $i \leq d$.

Proof: Since $\operatorname{gr}_{F}^{\bullet}(R)$ is a graded Poisson algebra containing $\operatorname{gr}_{F}^{0}(R)=A$, the universal property of $P(A)$ gives a morphism $P(A) \rightarrow \operatorname{gr}_{F}^{\bullet}(R)$ vanishing on $P^{\geq d+1}(A)$, so we get $\varphi$ : 
$P(A) / P^{\geq d+1}(A) \rightarrow \operatorname{gr}_{F}^{\bullet}(R)$. We will prove that $\varphi$ is an isomorphism. In fact, by induction we can assume that the homogeneous part $\varphi^{i}: P^{i}(A) \rightarrow \operatorname{gr}_{F}^{i}(R)$ is an isomorphism for $i<d$, so only $\varphi^{d}$ needs to be treated. Note that $\operatorname{gr}_{F}^{d}(R)=F^{d} R$ is a central ideal in $R$.

Let $x \in \operatorname{Spec}(A)$ be a C-point. For an $A$-module $N$ we denote $\left.N\right|_{x}=N / \mathbf{m}_{x, a b} N$ the fiber of $N$ at $x$. Here $\mathbf{m}_{x, a b} \subset A$ is the ideal of $x$. Since the source and the target of $\varphi^{d}$ are $A$-modules of finite type, it is enough to prove that for any $x$ as above the map of fibers $\left.\varphi^{d}\right|_{x}:\left.\left.P^{d}(A)\right|_{x} \rightarrow\left(F^{d} R\right)\right|_{x}$ is an isomorphism. Let $\left(R_{x}, \mathbf{m}\right)$ and $\left(A_{x}, \mathbf{m}_{a b}\right)$ be the localizations of $R$ and $A$ at $x$. Since $P^{d}$ and $\operatorname{gr}_{F}^{d}$ commute with localizations, we can identify $\left.\left.P^{d}(A)\right|_{x} \cong P^{d}\left(A_{x}\right)\right|_{x},\left.\left.\left(F^{d} R\right)\right|_{x} \cong\left(F^{d} R_{x}\right)\right|_{x}$. Consider the commutative diagram of $A_{x}$-modules

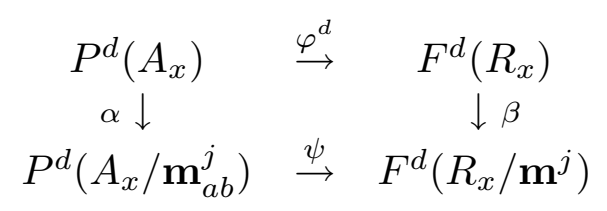

where $j \gg 0$, the maps $\alpha, \beta$ are induced by the functoriality of $P^{d}$ and $F^{d}$, and $\psi$ is defined similarly to $\varphi$, via the universal property of Poisson envelopes. We claim that for $j \gg 0$ the maps $\alpha, \beta, \psi$ give isomorphisms on fibers at $x$.

The fact that $\alpha$ gives an isomorphism follows from (4.1.3) and from an identification $A_{x} / \mathbf{m}_{a b}^{j} \simeq \mathbf{C}\left[x_{1}, \ldots, x_{n}\right] /\left(x_{1}, \ldots, x_{n}\right)^{j}$. Namely, we explicitly identify the Poisson envelope of the latter algebra as a quotient of $P\left(\mathbf{C}\left[x_{1}, \ldots, x_{n}\right]\right)=\mathcal{P}$ ois $\left(x_{1}, \ldots, x_{n}\right)$.

The fact that $\psi$ gives an isomorphism, follows from the identification

$$
R_{x} / \mathbf{m}^{j} \simeq \mathbf{C}\left\langle x_{1}, \ldots, x_{n}\right\rangle /\left(x_{1}, \ldots, x_{n}\right)^{j}+F^{d+1},
$$

see (1.5.1), and from the explicit description of $F^{\bullet} \mathbf{C}\left\langle x_{1}, \ldots, x_{n}\right\rangle$ given in (3.4.5).

Finally, we prove that $\beta$ gives an isomorphism on fibers. We have

$$
\begin{aligned}
\left.F^{d}\left(R_{x}\right)\right|_{x} & =F^{d}\left(R_{x}\right) / \mathbf{m} \cdot F^{d}\left(R_{x}\right) \\
\left.F^{d}\left(R_{x} / \mathbf{m}^{j}\right)\right|_{x} & =F^{d}\left(R_{x}\right) / \mathbf{m} \cdot F^{d}\left(R_{x}\right)+\mathbf{m}^{j} \cap F^{d}\left(R_{x}\right)
\end{aligned}
$$

So we are reduced to the next lemma.

(4.2.2) Lemma. If $j>d$, then $\mathbf{m}^{j} \cap F^{d}\left(R_{x}\right) \subset \mathbf{m} \cdot F^{d}\left(R_{x}\right)$.

Proof: Let $\left(\hat{R}_{x}, \hat{\mathbf{m}}\right)$ be the $m$-adic completion of $R$. By (1.5.1),

$$
\hat{R}_{x} \simeq \mathbf{C}\left\langle\left\langle x_{1}, \ldots, x_{n}\right\rangle\right\rangle / F^{d+1} .
$$

This is again a Noetherian local algebra. Now, the explicit description of $\mathbf{C}\left\langle\left\langle x_{1}, \ldots, x_{n}\right\rangle\right\rangle$ together with its NC-filtration given in (3.5.5), implies that $\hat{\mathbf{m}}^{j} \cap F^{d} \hat{R}_{x} \subset \hat{\mathbf{m}} \cdot F^{d} \hat{R}_{x}$ for $j>d$. Our statement would follow therefore from the equality

$$
\mathbf{m} \cdot F^{d}\left(R_{x}\right)=\left(\hat{\mathbf{m}} \cdot F^{d}\left(\hat{R}_{x}\right)\right) \cap R
$$

But this is a particular case of the following version of the Krull lemma whose proof is the same as in the classical case ([Eis], Ch. 4). 
(4.2.4) Lemma. Let $R$ be a (not necessarily commutative) Noetherian local ring, $\hat{R}$ is completion, $I \subset R$ a central ideal, $\hat{I}=I \hat{R}$. If $\hat{I}$ is central, then $\hat{I} \cap R=I$.

Theorem 4.2.1 is proved.

(4.2.5) Corollary. Let $R$ be a $d$-smooth algebra. Then $H_{2}\left(R, R_{a b}\right) \simeq Q_{R_{a b}}^{d+1}\left(\Omega_{R_{a b}}^{1}\right)$ is an $R_{a b}$-module.

(4.2.6) Remark. It would be interesting to describe $H_{i}\left(R, R_{a b}\right), i>2$ as certain explicit polynomial functors of $\Omega_{R_{a b}}^{1}$, generalizing the Hochschild-Kostant-Rosenberg theorem $H_{i}\left(R_{a b}, R_{a b}\right)=\Lambda^{i} \Omega_{R_{a b}}^{1}$. The analogous group-theoretical problem (find the integral homology of the free $(d+1)$-stage nilpotent group, see (1.6.4)) is quite difficult. Even rationally, when groups can be replaced by Lie algebras, the answer for the full homology has been found only for $d \leq 1$, see [Sig].

(4.3) Cohomological classification of thickenings. Let $M$ be a smooth algebraic variety and $X \supset M$ be a $d$-smooth thickening. For a Zariski open $U \subset \mathcal{M}$ let $U^{(d)}$ be the induced thickening of $U$. Let $\mathcal{T}_{X}(U)$ be the category whose objects are $(d+1)$-smooth thickenings $V \supset U^{(d)}$ and morphisms are morphisms of NC-schemes identical on $U^{(d)}$. Denote by $T_{M}$ the tangent sheaf of $M$.

(4.3.1) Proposition. (a) Each $\mathcal{T}_{X}(U)$ is a groupoid.

(b) The correspondence $U \mapsto \mathcal{T}_{X}(U)$ is a stack $\mathcal{T}_{X}$ of groupoids on the Zariski topology of $\mathcal{M}$.

(c) The stack $\mathcal{T}_{X}$ is a gerbe with band $\operatorname{Hom}\left(Q^{d+1} T_{M}, T_{M}\right)$.

Proof: (a) follows from (1.2.7). Part (b) is clear. Part (c) follows from (4.2.5) and (1.3.7).

By applying the Grothendieck-Giraud formalism of nonabelian cohomology via stacks [Bry], we get the following result.

(4.3.2) Theorem. Any $d$-smooth thickening $X \supset M$ defines a natural cohomology class (obstruction) $\gamma_{X} \in H^{2}\left(M, \operatorname{Hom}\left(Q^{d+1} T_{M}, T_{M}\right)\right) . \quad A(d+1)$-smooth thickening $Y \supset X$ exists if and only if $\gamma_{X}=0$. If this is the case, the set of such $Y$ modulo isomorphisms identical on $X$, is a principal homogeneous space over $H^{1}\left(M, \operatorname{Hom}\left(Q^{d+1} T_{M}, T_{M}\right)\right)$.

(4.4) Jet Bundles. Let $M$ be a smooth algebraic variety, $\operatorname{dim}(M)=n$. For $x \in M$ let $\mathbf{m}_{x, a b} \subset \mathcal{O}_{M}$ be the ideal of $M$. Denote also by $\mathbf{m}_{a b} \subset \mathbf{C}\left[x_{1}, \ldots, x_{n}\right]$ the ideal $\left(x_{1}, \ldots, x_{n}\right)$, and by $\mathbf{m}$ the similar ideal in $\mathbf{C}\left\langle x_{1}, \ldots, x_{n}\right\rangle$.

Let $l \geq 0$ and $J_{M}^{l}$ be the bundle (sheaf) of $l$-jets of functions on $M$. The fiber of $J_{M}^{l}$ at $x \in M$ is $\mathcal{O}_{M} / \mathbf{m}_{x, a b}^{l+1}$. Thus $J_{\mathcal{M}}^{l}$ is a sheaf of $\mathcal{O}_{\mathcal{M}}$-algebras locally isomorphic to $\mathcal{O}_{\mathcal{M}} \otimes\left(\mathbf{C}\left[x_{1}, \ldots, x_{n}\right] / \mathbf{m}_{a b}^{l+1}\right)$. Let $j_{l}^{a b}: \mathcal{O}_{M} \rightarrow J_{M}^{l}$ be the universal $l$ th order differential operator. The sections of $\operatorname{Im}\left(j_{l}^{a b}\right)$ will be called pure jets.

Let now $X$ be a $d$-smooth thickening of $\mathcal{M}$. For $x \in \mathcal{M}$ let $\mathbf{m}_{x} \subset \mathcal{O}_{X}$ be the preimage of $\mathbf{m}_{x, a b}$. We have then a bundle (locally free sheaf) $J_{X}^{l}$ on $M$ whose fiber at $x \in X$ is $\mathcal{O}_{X} / \mathbf{m}_{x}^{l+1}$. Again, we have a natural morphism of sheaves $j_{l}: \mathcal{O}_{X} \rightarrow J_{X}^{l}$, lifting $j_{l}^{a b}$. 
(4.4.1) Proposition. $J_{X}^{l}$ is a sheaf of $\mathcal{O}_{M}$-algebras locally isomorphic to

$$
\mathcal{O}_{M} \otimes\left(\mathbf{C}\left\langle x_{1}, \ldots, x_{n}\right\rangle /\left(\mathbf{m}^{l+1}+F^{d+1}\right)\right) .
$$

Its abelianization is identified with $J_{M}^{l}$.

Proof: follows from (1.5.1).

Let $U \subset M$ be Zariski open, and $U^{(d)} \subset X$ be the induced thickening. Define the following category $\mathcal{J}_{X}(U)$. Its objects are pairs $(\mathbf{O}, \psi)$, where $\mathbf{O}$ is a sheaf of $\mathcal{O}_{U}$-algebras locally isomorphic to $\mathcal{O}_{U} \otimes\left(\mathbf{C}\left\langle x_{1}, \ldots, x_{n}\right\rangle / \mathbf{m}^{d+2}\right)$, and $\psi: \mathbf{O} \rightarrow \mathcal{J}_{X}^{d+1}$ is a surjection of sheaves of algebras with kernel $F^{d+1} \mathbf{O}$. A morphism $(\mathbf{O}, \psi) \rightarrow\left(\mathbf{O}^{\prime}, \psi^{\prime}\right)$ is an isomorphism $f: \mathbf{O} \rightarrow \mathbf{O}^{\prime}$ of sheaves of algebras such that $\psi^{\prime} f=\psi$.

Note that if $\mathbf{O}$ is any sheaf of $\mathcal{O}_{U}$-algebras locally isomorphic to $\mathcal{O}_{U} \otimes\left(\mathbf{C}\left\langle x_{1}, \ldots, x_{n}\right\rangle / \mathbf{m}^{d+2}\right)$, then the images of $\mathcal{O}_{U} \otimes\left(\mathbf{m} / \mathbf{m}^{d+2}\right)$ under all such isomorphisms coincide and define a sheaf of ideals $\underline{\mathbf{m}} \subset \mathbf{O}$, with $\mathbf{O} / \underline{\mathbf{m}}=\mathcal{O}_{U}$. Define the category $\mathcal{A}_{X}(U)$ whose objects are sheaves of algebras $\mathbf{O}$ as before together with an isomorphism $\varphi: \mathbf{O} / \mathbf{m}^{d+1} \stackrel{\sim}{\rightarrow} J_{X}^{d}$ and morphisms defined in a similar way. We have the functors

$$
\mathcal{T}_{X}(U) \stackrel{e_{U}}{\longrightarrow} \mathcal{J}_{X}(\mathbf{U}) \stackrel{f_{U}}{\longrightarrow} \mathcal{A}_{X}(U)
$$

defined as follows. For a $(d+1)$-smooth thickening $V \supset U^{(d)}$ we set $e_{U}(V)=\mathcal{J}_{V}^{d+1}$. For an object $(\mathbf{O}, \psi)$ of $\mathcal{J}_{X}(U)$ we set $f_{U}(\mathbf{O}, \psi)=(\mathbf{O}, p \psi)$ where $p: J_{X}^{d+1} \rightarrow J_{X}^{d}$ is the natural surjection.

(4.4.2) Proposition. (a) The correspondences $U \mapsto \mathcal{J}_{X}(U), \mathcal{A}_{X}(U)$ form stacks of groupoids $\mathcal{J}_{X}, \mathcal{A}_{X}$ on $M$, and the functors $e_{U}, f_{U}$ define morphisms of stacks $\mathcal{T}_{X} \stackrel{e}{\rightarrow} \mathcal{J}_{X} \stackrel{f}{\rightarrow} \mathcal{A}_{X}$.

(b) The stack $\mathcal{J}_{X}$ is a gerbe with band $\operatorname{Hom}\left(Q^{d+1} T_{M}, T_{M}\right)$, the stack $\mathcal{A}_{X}$ is a gerbe with band $\operatorname{Hom}\left(T_{M}^{\otimes(d+1)}, T_{M}\right)$.

(c) The functor $e$ is an equivalence of gerbes with band $\operatorname{Hom}\left(Q^{d+1} T_{M}, T_{M}\right)$.

(d) The functor $f$ is compatible with the morphism

$$
\epsilon: \operatorname{Hom}\left(Q^{d+1} T_{M}, T_{M}\right) \rightarrow \operatorname{Hom}\left(T_{M}^{\otimes(d+1)}, T_{M}\right)
$$

induced by the embedding $Q^{d+1} \Omega_{M}^{1} \hookrightarrow\left(\Omega_{M}^{1}\right)^{\otimes d+1}$.

Proof: (a) is clear. Let us prove (b). Let first an object $(\mathbf{O}, \varphi)$ of $\mathcal{A}_{X}(U)$ be given. Then $\underline{\mathbf{m}} / \underline{\mathbf{m}}^{2} \simeq \Omega_{U}^{1}$ and thus $\underline{\mathbf{m}}^{i} / \underline{\mathbf{m}}^{i+1} \simeq\left(\Omega_{U}^{1}\right)^{\otimes i}$ for $i \leq d+1$, while $\underline{\mathbf{m}}^{d+2}=0$. Thus $\mathbf{O}$ is a central extension

$$
0 \rightarrow\left(\Omega_{M}^{1}\right)^{\otimes d+1} \rightarrow \mathbf{O} \stackrel{\varphi}{\rightarrow} \mathbf{O} / \underline{\mathbf{m}}^{d+1} \rightarrow 0
$$

Automorphisms of such an extension compatible with $\varphi$, form a Abelian group sheaf of Abelian groups identified with $\operatorname{Hom}\left(T_{m}^{\otimes d+1}, T_{M}\right)$, see (1.2.5). So $\mathcal{A}_{X}$ is a gerbe with band $\operatorname{Hom}\left(T_{M}^{\otimes(d+1)}, T_{M}\right)$. 
Further, an object $(\mathbf{O}, \psi)$ of $\mathcal{J}_{X}(U)$ gives a central extension

$$
0 \rightarrow F^{d+1} \mathbf{O} \rightarrow \mathbf{O} \stackrel{\psi}{\rightarrow} \mathcal{J}_{X}^{d+1} \rightarrow 0
$$

and our statement follows in the same way as above once we recall the interpretation of the functor $Q^{d+1}$ given in (3.4.6).

To prove (c) it is enough to show that $e$ is just a morphism of gerbes with the same band: it will then be automatically an equivalence. But this is clear from the definition of $e$ : given $\mathbf{O}=J_{U}^{d+1}$, the consideration of jets at any $x \in M$ gives a canonical identification $F^{d+1} \mathbf{O} \leftarrow F^{d+1} \mathbf{O}_{U}$. As for $(\mathrm{d})$, this just expresses the fact that for $(\mathbf{O}, \psi) \in \mathcal{J}_{X}(U)$ the embedding $\operatorname{Aut}(\mathbf{O}, \psi) \hookrightarrow \operatorname{Aut}(\mathbf{O}, p \psi)$ is precisely identified with the embedding

$$
\operatorname{Hom}\left(Q^{d+1} T_{M}, T_{M}\right) \rightarrow \operatorname{Hom}\left(T_{M}^{\otimes(d+1)}, T_{M}\right)
$$

mentioned in the statement. But this is also clear, since $F^{d+1} \mathbf{O} \subset \underline{\mathbf{m}}^{d+1}$ is precisely $Q^{d+1}\left(\Omega_{M}^{1}\right) \hookrightarrow\left(\Omega_{M}^{1}\right)^{\otimes(d+1)}$.

Let us reformulate part (c) of the above proposition.

(4.4.3) Corollary. In order to extend a given $d$-smooth thickening $X \supset M$ to a $(d+$ 1)-smooth thickening, it suffices to construct a sheaf $\mathbf{O}$ of $\mathcal{O}_{M}$-algebras on $M$ locally isomorphic to $\mathcal{O}_{M} \otimes\left(\mathbf{C}\left\langle x_{1}, \ldots, x_{n}\right\rangle / \mathbf{m}^{d+2}\right)$ such that $\mathbf{O} / F^{d+1} \mathbf{O} \simeq J_{X}^{d+1}$.

Note that in this case $\mathbf{O}_{a b} \simeq J_{M}^{d+1}$, and the structure sheaf $\mathcal{O}_{Y}$ of the thickening consists of those sections $g$ of $\mathbf{O}$ for which $g_{a b}$ is a pure jet.

Let $\mathcal{A} s s\left(\Omega_{M}^{1}\right)$ be the tensor algebra of $\Omega_{M}^{1}$ over $\mathcal{O}_{M}$ and $\mathcal{A} s s^{\leq d}\left(\Omega_{M}^{1}\right)$ its quotient obtained by disregarding the homogeneous components of degree $>d$. Let $\mathcal{G}_{d}$ be the sheaf of groups on $M$ formed by algebra automorphisms of $\mathcal{A} s s^{\leq d}\left(\Omega_{M}^{1}\right)$ whose action on $\Omega_{M}^{1}$ is identical modulo $\left(\Omega_{M}^{1}\right)^{\otimes i}, i \geq 2$. Similarly, let $S \leq d\left(\Omega_{M}^{1}\right)$ be the truncated symmetric algebra and $\mathcal{G}_{d}^{\prime}$ be the sheaf of automorphisms of $S^{\leq d}\left(\Omega_{M}^{1}\right)$ identical modulo terms of degree $\geq 2$. Clearly we have a surjective homomorphism $\sigma_{d}: \mathcal{G}_{d} \rightarrow \mathcal{G}_{d}^{\prime}$. The next proposition is straightforward.

(4.4.4) Proposition. (a) $\mathcal{G}_{d}$ and $\mathcal{G}_{d}^{\prime}$ are sheaves of nilpotent groups on $M$ fitting into short exact sequences with central kernels:

$$
\begin{gathered}
1 \rightarrow \operatorname{Hom}\left(T_{M}^{\otimes d+1}, T_{M}\right) \rightarrow \mathcal{G}_{d+1} \rightarrow \mathcal{G}_{d} \rightarrow 1 \\
1 \rightarrow \operatorname{Hom}\left(S^{d+1} T_{M}, T_{M}\right) \rightarrow \mathcal{G}_{d+1}^{\prime} \rightarrow \mathcal{G}_{d}^{\prime} \rightarrow 1 .
\end{gathered}
$$

(b) $H^{1}\left(M, \mathcal{G}_{d}\right)$ is identified with the set of isomorphism classes of sheaves of algebras $\mathbf{O}$ on $M$ locally isomorphic to $\mathcal{O}_{M} \otimes\left(\mathbf{C}\left\langle x_{1}, \ldots, x_{n}\right\rangle / \mathbf{m}^{d+1}\right)$, equipped with an identification $\zeta: \underline{\mathbf{m}} / \underline{\mathbf{m}}^{2} \rightarrow \Omega_{M}^{1}$ (modulo isomorphisms preserving these identifications). 
(c) Similarly, $H^{1}\left(M, \mathcal{G}_{d}^{\prime}\right)$ is identified with the set of isomorphism classes of sheaves $\mathbf{O}^{\prime}$ of $\mathcal{O}_{M}$-algebras locally isomorphic to $\mathcal{O}_{M} \otimes\left(\mathbf{C}\left[x_{1}, \ldots, x_{n}\right] / \mathbf{m}_{a b}^{d+1}\right)$ equipped with an identification $\underline{\mathbf{m}}_{a b} / \underline{\mathbf{m}}_{a b}^{2} \simeq \Omega_{M}^{1}$.

Note, in particular, that the sheaf $J_{M}^{d}=\mathbf{O}^{\prime}$ satisfies the conditions of (c) so gives same class $\alpha_{d, M} \in H^{1}\left(M, \mathcal{G}_{d}^{\prime}\right)$. Further, any $d$-smooth thickening $X \supset M$ gives the sheaf $J_{X}^{d}=\mathbf{O}$ satisfying the conditions of (b), so gives a class $\alpha_{d, X} \in H^{1}\left(M, \mathcal{G}_{d}\right)$ such that $\sigma_{d}\left(\alpha_{d, X}\right)=\alpha_{d, M}$.

(4.4.5) Proposition. If $X$ is a $d$-smooth thickening of $M$, then the image of the obstruction $\gamma_{X} \in H^{2}\left(M, H o m\left(Q^{d+1} T_{M}, T_{M}\right)\right)$ under

$$
\epsilon_{*}: H^{2}\left(M, \operatorname{Hom}\left(Q^{d+1} T_{M}, T_{M}\right)\right) \rightarrow H^{2}\left(M, \operatorname{Hom}\left(T_{M}^{\otimes(d+1)} T_{M}, T_{M}\right)\right)
$$

is equal to $\delta\left(\alpha_{d, X}\right)$ where $\delta$ is the nonabelian coboundary map associated to (4.4.4.1).

Proof: This is a consequence of 4.4.2(d).

(4.4.6) Proposition. The map $\epsilon$ from 4.4.2(d) is the embedding of a direct summand. In particular the induced map $\epsilon_{*}$ on the cohomology is injective.

Proof: For any $d$ the value of the polynomial functor $Q^{d}$ on a vector space $V$ can be described as

$$
Q^{d}(V)=\left(V^{\otimes d} \otimes W_{d}\right)^{S_{d}}
$$

where $W_{d}$ is a certain representation of the symmetric group $S_{d}$. The embedding $Q^{d}(V) \subset$ $V^{\otimes d}$ corresponds to an embedding $W_{d} \subset \mathbf{C}\left[S_{d}\right]$ of $W_{d}$ into the regular representation. Now taking the complement $W_{d}^{\prime}$ to $W_{d}$ in $\mathbf{C}\left[S_{d}\right]$ and defining $R^{d}(V)=\left(V^{\otimes d} \otimes W_{d}^{\prime}\right)^{S_{d}}$, we find that $V^{\otimes d}=Q^{d}(V) \oplus R^{d}(V)$ as $G L(V)$-modules. Thus $\left(\Omega_{M}^{1}\right)^{\otimes d} \simeq Q^{d}\left(\Omega_{M}^{1}\right) \oplus R^{d}\left(\Omega_{M}^{1}\right)$ as vector bundles, whence the statement.

(4.5) First order thickenings and Atiyah classes. Let $M$ be as before. Among 1smooth thickenings of $M$ there is a distinguished one $X_{0}$, with $\mathcal{O}_{X_{0}}=\mathcal{O}_{M} \oplus \Omega_{M}^{2}$ and the multiplication given in (1.3.9). Recalling that the functor $Q^{1}=\Lambda^{2}$ is the second exterior power, we get from (4.3.2) the following.

(4.5.1) Proposition. The set of isomorphism classes of 1-smooth thickenings of $M$ is canonically identified with $H^{1}\left(M, \Omega_{M}^{2} \otimes T_{M}\right)$, so that $X_{0}$ corresponds to 0 .

We denote by $\alpha_{X}^{-} \in H^{1}\left(M, \Omega_{M}^{2} \otimes T_{M}\right)$ the class corresponding to a thickening $X$. Recall (4.4) that we have the classes

$$
\begin{aligned}
\alpha_{2, X} \in H^{1}\left(\mathcal{M}, \mathcal{G}_{2}\right) & =H^{1}\left(\mathcal{M}, \operatorname{Hom}\left(T_{M}^{\otimes 2}, T_{M}\right)\right), \\
\alpha_{2, M} \in H^{1}\left(\mathcal{M}, \mathcal{G}_{2}^{\prime}\right) & =H^{1}\left(\mathcal{M}, \operatorname{Hom}\left(S^{2} T_{M}, T_{M}\right)\right)
\end{aligned}
$$

classifying the bundles $\mathcal{J}_{X}^{2}, \mathcal{J}_{M}^{2}$. In fact, $\alpha_{2, M}$ is the Atiyah class of the tangent bundle of $M$, see [Kap]. So we call $\alpha_{2, X}$ the NC-Atiyah class of $X$. 
(4.5.2) Proposition. With respect to the natural identification

$$
H^{1}\left(M, \operatorname{Hom}\left(T_{M}^{\otimes 2}, T_{M}\right)\right)=H^{1}\left(M, \operatorname{Hom}\left(S^{2} T_{M}, T_{M}\right)\right) \oplus H^{1}\left(M, \Omega_{M}^{2} \otimes T_{M}\right)
$$

we have $\alpha_{2, X}=\alpha_{2, M} \oplus \alpha_{X}^{-}$.

Proof: $\Omega_{M}^{2} \otimes T_{M}$ is the sheaf of automorphisms of $X_{0}$ identical on $M$. The identification of (4.5.1) is obtained explicitly by identifying, for any affine $U \subset M$, the induced thickening $U^{(1)} \subset X$ with the trivial thickening $U_{0} \subset X_{0}$ and looking at the discrepancies over the intersections which give a Čech 1-cocycle in $\Omega_{M}^{2} \otimes T_{M}$ representing $\alpha_{X}^{-}$. Similarly, $\alpha_{2, X}$ is obtained by identifying $\left.J_{X}^{2}\right|_{U}$ with $\mathcal{A} s s^{\leq 2}\left(\Omega_{U}^{1}\right)$ by an algebra automorphism with identical linear part, and then taking the discrepancies.

Notice now that $\mathcal{A} s s^{\leq 2}\left(\Omega_{U}^{1}\right)=S^{\leq 2}\left(\Omega_{U}^{1}\right) \oplus \Omega_{U}^{2}$. So if it is identified with $\left.J_{X}^{2}\right|_{U}$, then $\mathcal{O}_{U^{(1)}}$ (realized inside $\left.J_{X}^{2}\right|_{U}$ as the sheaf of sections whose image in $J_{U}^{2}$ is a pure jet) becomes explicitly identified with the direct sum of $\mathcal{O}_{U} \subset J_{\mathbf{U}}^{2} \simeq S^{\leq 2}\left(\Omega_{U}^{1}\right)$ and $\Omega_{U}^{2}$. In other words, $U^{(1)}$ becomes identified with $U_{0}$. This shows that the antisymmetrization of $\alpha_{2, X}$ is $\alpha_{X}^{-}$, proving the proposition.

(4.5.3) Theorem. Let $X$ be a 1-smooth thickening of $M$ and

$$
\alpha=\alpha_{2, X} \in H^{1}\left(M, \operatorname{Hom}\left(T_{M}^{\otimes 2}, T_{M}\right)\right)
$$

be its NC-Atiyah class. Then $X$ extends to a second order thickening if and only if $\alpha$ satisfies the anti-associativity condition:

$$
\alpha \circ(\alpha \otimes 1)=-\alpha \circ(1 \otimes \alpha) \quad \text { in } \quad H^{2}\left(M, \operatorname{Hom}\left(T_{M}^{\otimes 3}, T_{M}\right)\right) .
$$

Proof: The sheaf $\mathcal{G}_{2}$ is a central extension

$$
1 \rightarrow \operatorname{Hom}\left(T_{M}^{\otimes 3}, T_{M}\right) \rightarrow \mathcal{G}_{2} \rightarrow \operatorname{Hom}\left(T_{M}^{\otimes 2}, T_{M}\right) \rightarrow 0 .
$$

By (4.4.5-6) the extension of $X$ to a 2-smooth thickening is possible if and only if $\delta(\alpha)=0$, where $\delta$ is the coboundary map of (4.5.4). So we need to prove that

$$
\delta(\alpha)=\alpha \circ(\alpha \otimes 1)+\alpha \circ(1 \otimes \alpha) .
$$

For this, let us describe $\mathcal{G}_{2}$ explicitly as follows.

(4.5.6) Lemma. As a sheaf of sets,

$$
\mathcal{G}_{2} \simeq \operatorname{Hom}\left(T_{M}^{\otimes 2}, T_{M}\right) \times \operatorname{Hom}\left(T_{M}^{\otimes 3}, T_{M}\right) .
$$

Under this identification, the product of two sections $\varphi=\left(\varphi_{2}, \varphi_{3}\right)$ and $\psi=\left(\psi_{2}, \psi_{3}\right)$, is given by

$$
\begin{aligned}
\varphi \psi & =\left(\varphi_{2}+\psi_{2}, \varphi_{3}+\psi_{3}+\varphi_{2} \circ\left(1 \otimes \psi_{2}+\psi_{2} \otimes 1\right)\right), \text { and } . \\
\varphi^{-1} & =\left(-\varphi_{2},-\varphi_{3}+\varphi_{2} \circ\left(1 \otimes \varphi_{2}+\varphi_{2} \otimes 1\right)\right)
\end{aligned} .
$$


Proof: The identification is obtained first for the steaf of Lie algebras corresponding to $\mathcal{G}_{2}$, by using the $G L\left(T_{M}\right)$-action and then translated to $\mathcal{G}_{2}$ via the exponential map. To see that the multiplication has precisely this form, it is enough to consider just one vector space $T$ and the group $G_{2}$ of automorphisms of $\mathcal{A} s s^{\leq 3}\left(T^{*}\right)$ with identical linear part. In coordinates $x_{1}, \ldots, x_{n}$ an element of $G_{2}$ can be written as

$$
x_{i} \mapsto x_{i}+\sum_{j, k} a_{i}^{j k} x_{j} x_{k}+\sum_{p, q, r} a_{i}^{p q r} x_{p} x_{q} x_{r}
$$

where $\left\|a_{i}^{j k}\right\|$ form $\varphi_{2} \in \operatorname{Hom}\left(T^{\otimes 2}, T\right)$ and $\left\|a_{i}^{p q r}\right\|$ form $\varphi_{3} \in \operatorname{Hom}\left(T^{\otimes 3}, T\right)$. Our lemma is obtained by writing the composition of two such changes of variables in the tensor notation.

To prove (4.5.5), let $\left\{U_{i}\right\}$ be a Zariski open covering of $\mathcal{M}$ and $\Phi=\left(\varphi_{2}^{(i j)}\right)$ be a Čech 1-cocycle in $\operatorname{Hom}\left(T_{M}^{\otimes 2}, T_{M}\right)$ representing $\alpha$. To find the 2-cocycle $\psi=\delta(\Phi)$, we need to lift each $\varphi_{2}^{(i j)}$ to a section $\varphi^{(i j)}$ of $\mathcal{G}_{2}$ and set

$$
\psi^{(i j k)}=\left(\varphi^{(i k)}\right)^{-1} \varphi^{(j k)} \varphi^{(i j)} .
$$

Because of the above lemma, we can take $\varphi^{(i j)}=\left(\varphi_{2}^{(i j)}, 0\right)$ and then an immediate calculation gives

$$
\psi^{(i j k)}=\left(0, \varphi_{2}^{(j k)} \circ\left(1 \otimes \varphi^{(i j)}+\varphi^{(i j)} \otimes 1\right)\right)
$$

which is the Alexander-Whitney product of $\Phi$ and $(1 \otimes \Phi+\Phi \otimes 1)$. Theorem is proved.

The anti-associativity property becomes ordinary associativity under suspension.

(4.5.7) Corollary. Let $X$ be a 1-smooth thickening of $\mathcal{M}$ extendable to second order, and $\alpha=\alpha_{2, X}$. Let $\mathcal{A}$ be a sheaf of commutative $\mathcal{O}_{M}$-algebras. Then the operation

$$
H^{i}\left(M, \mathcal{A} \otimes T_{M}\right) \otimes H^{j}\left(M, \mathcal{A} \otimes T_{M}\right) \stackrel{\cup}{\rightarrow} H^{i+j}\left(M, \mathcal{A} \otimes T_{M}^{\otimes 2}\right){\stackrel{(-1)^{i}}{\rightarrow}}^{i} H^{i+j+1}\left(M, \mathcal{A} \otimes T_{M}\right)
$$

makes $H^{\bullet-1}\left(M, \mathcal{A} \otimes T_{M}\right)$ into a graded associative algebra. When $\mathcal{A}=\mathcal{O}_{M}$, this algebra is graded commutative.

Proof. The associativity follows by direct checking of signs, using the Koszul rules. The graded Lie algebra associated to $H^{\bullet-1}\left(M, \mathcal{A} \otimes T_{M}\right)$ is clearly induced by the symmetrization of $\alpha$, which is $\alpha_{2, M}$. This graded Lie algebra structure was studied in [Kap], where it was proved that for $\mathcal{A}=\mathcal{O}_{M}$ the bracket vanishes.

(4.6) Operadic interpretation of the obstruction via Stasheff polytopes. We now give a conceptual interpretation of the obstruction $\gamma_{X}$ from Theorem 4.3.2, for arbitrary d. For this, we will use the language of operads, see, e.g., [GK1] [GK2], and follow the conventions of $[\mathrm{Kap}]$, §3. In particular, by $\Sigma \mathcal{P}$ we denote the suspension of a dg-operad $\mathcal{P}$.

Given a graded operad $\mathcal{P}$, an element $\xi \in \mathcal{P}(2)$ of degree 0 will be called associative, if $\xi(\xi, 1)=\xi(1, \xi)$ in $\mathcal{P}(3)$. 
Let $M$ be a smooth algebraic variety, $T=T_{M}$. The sheaves $\mathcal{E}_{T}(n)=\operatorname{Hom}\left(T^{\otimes n}, T\right)$ form a sheaf $\mathcal{E}_{T}$ of operads on $\mathcal{M}$. Thus the $\mathbf{E}_{T}(n)=H^{\bullet}\left(M, \mathcal{E}_{T}(n)\right.$ form a graded operad $\mathbf{E}_{T}$. Given a 1-smooth thickening $X \supset M$, it NC-Atiyah class $\alpha=\alpha_{2, X}$ is an element of $\mathbf{E}_{T}(2)$ of degree 1. Thus the de-suspension $\Sigma^{-1}(\alpha) \in\left(\Sigma^{-1} \mathbf{E}_{T}\right)(2)$ is an element of degree 0. Theorem 4.5.3 can be formulated as follows.

(4.6.1) Corollary. $X$ can be extended to a 2-smooth thickening if and only if $\Sigma^{-1}(\alpha)$ is an associative element.

We now construct a $d g$-model for $\mathbf{E}_{T}$. Fix an affine covering $\left\{U_{i}\right\}$ of $M$, with intersections $U_{i_{o} \cdots i_{m}}$ affine as well. Let $j_{i_{o} \cdots i_{m}}: U_{i_{o} \cdots i_{m}} \hookrightarrow M$ be the embedding and

$$
\check{\mathcal{C}}^{m}=\bigoplus_{i_{o} \cdots i_{m}}\left(j_{i_{o} \cdots i_{m}}\right)_{*} j_{i_{o} \cdots i_{m}}^{*}\left(\mathcal{O}_{M}\right), \quad \check{C}^{m}=\Gamma\left(M, \check{\mathcal{C}}^{m}\right)
$$

so that $\check{C}^{m}$ is the $m$-th term of the Čech resolution of $\mathcal{O}_{M}$. The $\check{\mathcal{C}}^{m}$ form a cosimplicial sheaf $\check{\mathcal{C}}^{\bullet}$ of commutative algebras on $M$. Let $\mathcal{C}^{\bullet}$ be the sheaf of commutative $d g$-algebras obtained from $\check{\mathcal{C}}^{\bullet}$ by the Thom-Sullivan construction $[\mathrm{HS}]$. For a quasicoherent sheaf $\mathcal{F}$ on $M$ let

$$
\mathcal{C}^{\bullet}(\mathcal{F})=\mathcal{C}^{\bullet} \otimes \mathcal{F}, \quad C^{\bullet}(\mathcal{F})=\Gamma(M, \mathcal{C} \bullet(\mathcal{F})) .
$$

The following is then standard.

(4.6.2) Proposition. (a) $\mathcal{C}^{\bullet}$ is flat over $\mathcal{O}_{M}$.

(b) For every $\mathcal{F}$ as above the $\mathcal{C}^{m}(\mathcal{F})$ have no higher cohomology. In particular, $C^{\bullet}(\mathcal{F})$ calculates $H^{\bullet}(M, \mathcal{F})$.

(c) There are natural morphisms of complexes

$$
C^{\bullet}\left(\mathcal{F}_{1}\right) \otimes C^{\bullet}\left(\mathcal{F}_{2}\right) \rightarrow C^{\bullet}\left(\mathcal{F}_{1} \otimes \mathcal{F}_{2}\right)
$$

compatible with permutations of tensor factors.

It follows from (c) that the complexes $C^{\bullet}\left(\mathcal{E}_{T}(n)\right)$ form a $d g$-operad $C^{\bullet} \mathcal{E}_{T}$ whose cohomology operad is $\mathbf{E}_{T}$.

Each time when we have a $d g$-operad $\mathcal{P}$ and an associative element $\xi \in H^{0} \mathcal{P}(2)$, we can ask, following Stasheff [Sta], about a hierarchy of finer properties on the level of cochains. Namely, if $m_{2} \in \mathcal{P}(2)^{0}$ is a cocycle representing $\xi$, and $m_{3} \in \mathcal{P}(3)^{-1}$ satisfies

$$
\partial m_{3}=m_{2}\left(m_{2}, 1\right)-m_{2}\left(1, m_{2}\right)
$$

(it exists because of the associativity of $\xi$ ), the element

$$
m_{3}\left(1,1, m_{2}\right)+m_{3}\left(m_{2}, 1,1\right)-m_{2}\left(1, m_{3}\right)-m_{3}\left(1, m_{2}, 1\right)-m_{2}\left(m_{3}, 1\right) \in \mathcal{P}(4)^{-1}
$$

is a cocycle. If we visualize $m_{2}$ as a binary operation $(a, b) \mapsto a b$ and $m_{3}$ as an edge (homotopy) connecting $a(b c)$ and $(a b) c$, then (4.6.3) represents the boundary of the MacLaneStasheff pentagon whose vertices correspond to the parenthesizings of abcd. More generally, we have the following definition [Sta]. 
(4.6.4) Definition. Let $\mathcal{P}$ be a $d g$-operad, $\xi \in H^{0} \mathcal{P}(2)$ an associative element. We say that $\xi$ is an $A_{d}$-element if there exist $m_{i}, \in \mathcal{P}(i)^{2-i}, 2 \leq i \leq d$ such that $m_{2}$ is a cocycle representing $\xi$, and for each $\nu \in[2, d]$, we have

$$
\partial m_{\nu}=\sum_{\substack{r+s=\nu+1 \\ r, s \geq 2}} \sum_{1 \geq k \geq r}(-1)^{k(s+1)} m_{r} \underbrace{(1, \ldots, 1}_{k-1}, m_{s}, 1, \ldots, 1) .
$$

Such a system $\left(m_{2}, \ldots m_{d}\right)$ will be called an $A_{d}$-structure on $\xi$.

Geometrically, the expression on the right represents the boundary of the Stasheff polytope, see [Sta].

We apply this concept to $\mathcal{P}=\Sigma^{-1} C^{\bullet} \mathcal{E}_{T}$.

(4.6.5) Theorem. Let $M$ be a smooth algebraic variety, $X \supset M$ a d-smooth thickening and $\alpha \in H^{1}\left(M, \operatorname{Hom}\left(T_{M}^{\otimes 2}, T_{M}\right)\right)$ the NC-Atiyah class of $X^{\leq 1}$. Then:

(a) $\Sigma^{-1}(\alpha)$ is an $A_{d+1}$-element.

(b) The image of the obstruction $\gamma_{X}$ in $H^{2}\left(M, \operatorname{Hom}\left(T_{M}^{\otimes(d+2)}, T_{M}\right)\right)$, see (4.4.5), is the cohomology class of the cocycle

$$
\left.\Sigma(\sum_{\substack{r+s=d+2 \\ 1 \leq k \leq r}}(-1)^{k(s+1)} m_{r} \underbrace{(1, \ldots, 1}_{k-1}, m_{s}, 1, \ldots, 1)\right) \in C^{2}\left(\operatorname{Hom}\left(T_{M}^{\otimes(d+2)}, T_{M}\right)\right)
$$

where $\left(m_{2}, \ldots, m_{d+1}\right)$ is an appropriate $A_{d+1}$-structure on $\Sigma^{-1}(\alpha)$.

Proof: It is an observation of Stasheff [Sta] that an $A_{d}$-structure on a monoid $G$ is precisely the data needed to build the classifying space of $G$ up to the $d$ th level. We apply this to the situation when $G$ is the shifted complex $\left(\mathcal{C}^{\bullet-1}\left(\mathrm{~T}_{M}\right), \partial\right)$ of sheaves of $\mathcal{O}_{M}$-modules, the operation is given by a 1-cocycle of $m_{2}$ representing $\alpha$ and the skeleton of the classifying space is the sheaf of graded algebras $\mathcal{C} \bullet\left(\mathcal{A} s s^{\leq d}\left(\Omega_{M}^{1}\right)\right)$ with an appropriate differential.

So we consider the sheaf $\mathcal{A} s s^{\leq(d+1)}\left(\Omega_{M}^{1}\right)$ of $\mathcal{O}_{M}$-algebras, filtered by the powers $\underline{\mathbf{m}}^{i}$. Accordingly, $\mathcal{C}^{\bullet}\left(\mathcal{A} s s^{\leq(d+1)}\left(\Omega_{M}^{1}\right)\right)$ is a sheaf of graded, filtered $\mathcal{C}^{\bullet}\left(\mathcal{O}_{M}\right)$-algebras.

(4.6.6) Proposition. $A_{d+1}$-sequences $\left(m_{2}, \ldots, m_{d+1}\right), m_{i} \in C^{1} \operatorname{Hom}\left(T_{M}^{\otimes(i)}, T_{M}\right)$, are in bijection with algebra differentials $\mathcal{D}$ of $\mathcal{C}^{\bullet}\left(\mathcal{A} s s^{\leq(d+1)}\left(\Omega_{M}^{1}\right)\right)$ of degree 1 , satisfying the following conditions:

(1) $\mathcal{D}(a m)=\partial(a) m+(-1)^{\operatorname{deg}(a)} a \cdot \mathcal{D}(m), \quad \mathrm{a} \in \mathcal{C}^{\bullet}\left(\mathcal{O}_{M}\right), m \in \mathcal{C}^{\bullet}\left(\mathcal{A} s s^{\leq}(\mathrm{d}+1)\left(\Omega_{M}^{1}\right)\right)$.

(2) $\mathcal{D}$ preserves the filtration and $\mathcal{D} \equiv \partial \otimes 1$ on the associated graded factors.

(3) $\mathcal{D}^{2}=0$.

Proof: An algebra derivation $\mathcal{D}$ satisfying (1-2), is uniquely defined by its restriction on $\Omega_{M}^{1}$, which gives morphisms $\tilde{m}_{i}: \Omega_{M}^{1} \rightarrow \mathcal{C}^{1}\left(\left(\Omega_{M}^{1}\right)^{\otimes i}\right)$ or, equivalently,

$$
m_{i} \in \mathcal{C}^{1}\left(\operatorname{Hom}\left(T_{M}^{\otimes i}, T_{M}\right)\right), i=2, \ldots, d+1 .
$$

The condition that $\left(m_{2}, \ldots, m_{d+1}\right)$ form an $A_{d+1}$-sequence, is equivalent to $\mathcal{D}^{2}=0$. Proposition is proved. 
(4.6.7) Proposition. Given a derivation $\mathcal{D}$ of $\mathcal{C}^{\bullet}\left(\mathcal{A} s s^{\leq(d+1)}\left(\Omega_{\mathrm{M}}^{1}\right)\right)$, the subsheaf $\operatorname{Ker}(\mathcal{D})$ $\subset \mathcal{C}^{0}\left(\mathcal{A s s}^{\leq} \leq{ }^{(d+1)}\left(\Omega_{M}^{1}\right)\right)$ is a sheaf of $\mathcal{O}_{\mathrm{M}}$-algebras locally isomorphic to $\mathcal{O}_{M} \otimes\left(\mathbf{C}\left\langle x_{1}, \ldots, x_{n}\right\rangle / \mathbf{m}^{d+2}\right)$ and equipped with an identification $\underline{\mathbf{m}} / \underline{\mathbf{m}}^{2} \simeq \Omega_{M}^{1}$.

Proof: Recalling the definition of $\mathcal{C}^{\bullet}$ as the Thom-Sullivan construction applied to the Čech complex, we find that $\operatorname{Ker}(\mathcal{D})$ is obtained by gluing the sheaves of algebras $\mathcal{A s s} \leq(d+1)\left(\Omega_{U_{i}}^{1}\right)$ according to a system of descent data.

Conversely, given a sheaf $\mathbf{O}$ of $\mathcal{O}_{M}$-algebras as in (4.6.7), we identify $\left.\mathbf{O}\right|_{U_{i}} \tilde{\rightarrow} \mathcal{A} s s^{\leq(d+1)}$ $\left(\Omega_{U_{i}}^{1}\right)$ by isomorphisms identical on $\underline{\mathbf{m}} / \underline{\mathbf{m}}^{2}$ and get a derivation $\mathcal{D}$. Proposition is proved.

This proves part (a) of Theorem 4.6.5. Further, this proves that whenever the cocycle in (b) is a coboundary, we can construct a sheaf $\mathbf{O}^{(\mathrm{d}+1)}$ on $M$ with $\mathbf{O}^{(d+1)} / m^{d+2} \simeq J_{\mathrm{X}}^{d}$. So the vanishing of $\varepsilon_{*}\left(\gamma_{X}\right)$ is equivalent to the vanishing of the cohomology class of the cocycle in (b). Further, this observation can be upgraded to the equality of the two cohomology classes, if we use once again the language of stacks. More precisely, $\varepsilon_{*}\left(\gamma_{X}\right)$ is represented, according to $(4.4 .2)(\mathrm{d})$, by the stack of extensions

$$
0 \rightarrow\left(\Omega_{X}^{1}\right)^{\otimes(d+2)} \rightarrow \mathbf{O}^{(d+1)} \rightarrow \mathrm{J}_{X}^{d} \rightarrow 0
$$

If we represent $J_{X}^{d}$ as $\operatorname{Ker}(\mathcal{D}) \subset \mathcal{C}^{0}\left(\mathcal{A s s}^{\leq}{ }^{(d+1)}\left(\Omega_{M}^{1}\right)\right)$ with $\mathcal{D}$ given by $\left(m_{2}, \ldots m_{d+1}\right)$, then the cocycle in (4.6.5)(b) is just $\tilde{\mathcal{D}}^{2}$, where $\tilde{\mathcal{D}}$ is the natural extension of $\mathcal{D}$ to $C^{\bullet}\left(\mathcal{A s s}^{\leq(d+2)}\left(\Omega_{M}^{1}\right)\right)$, obtained by taking $\tilde{m}_{d+2}=0$, see the proof of (4.6.6). For any Zariski open $U \subset M$ and any $\mu_{d+2} \in C^{1}\left(\operatorname{Hom}\left(T_{U}^{\otimes(d+2)}, T_{U}\right)\right)$ such that $\partial \mu_{d+2}$ equals the restriction of the cocycle in (4.6.5)(b) to $U$, the sequence $\left(m_{2}, \ldots, m_{d+1}, \mu_{d+2}\right)$ is an $A_{d+2}$-sequence and thus determines an extension (4.6.8) over $U$.

On the other hand, for any coherent sheaf $\mathcal{F}$ on $M$ and any cocycle $c \in C^{2}(\mathcal{F})=$ $\Gamma\left(M, \mathcal{C}^{2}(\mathcal{F})\right)$ the class $[c] \in H^{2}(M, \mathcal{F})$ can be represented by the following stack $(\mathcal{F}$ gerbe) $\mathcal{S}_{\mathrm{c}}$. The object of $\mathcal{S}_{c}(U), U \subset M$, are sections of $\left.\left.\partial^{-1}(c)\right|_{U} \subset \mathcal{C}^{1}(\mathcal{F})\right|_{\mathrm{U}}$. A morphism from $b$ to $b^{\prime}$, where $b, b^{\prime} \in \mathrm{Ob}\left(\mathcal{S}_{c}(U)\right)$, are sections $\alpha \in \Gamma\left(U, \mathcal{C}^{0}(\mathcal{F})\right)$ such that $\partial(a)=b-b^{\prime}$.

Now, taking for $c$ the cocycle from (4.6.5)(b), we find that the above reasoning defines in fact an equivalence of $\operatorname{Hom}\left(\mathrm{T}_{M}^{\otimes(d+2)}, T_{M}\right)$-gerbes between $\mathcal{S}_{c}$ and the gerbe of extensions (4.6.8). Theorem 4.6.5 is proved.

(4.6.9) Remarks. Let $X$ be a $d$-smooth thickening of $M$. It is natural to call a (left) vector bundle on $X$ a locally free sheaf $\mathcal{E}$ of left $\mathcal{O}_{X}$-modules. Such a sheaf defines a vector bundle $\mathcal{E}_{a b}$ on $M$ in the usual sense. Given a vector bundle $E$ on $M$, the problem of extending $E$ to a bundle $\mathcal{E}$ on $X$ (with $\mathcal{E}_{a b}=E$ ) can be analyzed in a way entirely parallel to the above. Thus, $E$ defines the Atiyah class

$$
\alpha_{E} \in H^{1}(M, \operatorname{Hom}(T \otimes E, E)),
$$

see, e.g., [Kap]. The condition that $E$ extends to a bundle on $X^{\leq 1}$, is:

$$
\alpha_{E} \circ\left(\alpha_{X} \otimes 1\right)=-\alpha_{E} \circ\left(1 \otimes \alpha_{E}\right) \quad \text { in } \quad H^{2}\left(M, \operatorname{Hom}\left(T_{M} \otimes T_{M} \otimes E, E\right),\right.
$$


where $\alpha_{X}=\alpha_{2, X}$ is the NC-Atiyah class of $X \leq 1$. After suspension, this becomes the associativity condition for a (left) module over an algebra, thus fitting nicely with (4.5.3) which gives the associativity condition for an algebra. The obstructions to extending $E$ to bundles on the further $X^{\leq i}$ can be interpreted in a similar way, using the concept of a left $A_{i}$-module over an $A_{i}$-algebra. We leave this to the reader. 


\section{$\S 5$. Examples of NC-manifolds.}

(5.1) The projective space. We define the NC-manifold $P_{\text {left }}^{n}=\left(P^{n}, \mathcal{O}_{\text {left }}^{\mathrm{NC}}\right)$ (the left NC-projective space) by gluing $n+1$ copies $U_{i}, i=0, \ldots, n$, of $A_{\mathrm{NC}}^{n}$. Namely, let $x_{j}^{(i)}, j \neq i$, be the coordinates in $U_{i}$, so that $U_{i}=\operatorname{Spf} \mathbf{C}\left\langle x_{j}^{(i)}\right\rangle_{\llbracket a b \rrbracket}$. We now use the noncommutative variables $t_{0}, \ldots, t_{n}$ to relate the $x_{j}^{(i)}$ together via

$$
x_{j}^{(i)}=t_{j} t_{j}^{-1}
$$

which gives the transition functions

$$
x_{j}^{(k)}=\left\{\begin{array}{l}
x_{j}^{(i)}\left(x_{k}^{(i)}\right)^{-1}, \quad j \neq i \\
\left(x_{k}^{(i)}\right)^{-1}, \quad j=i
\end{array}\right.
$$

These expressions should be understood as commutator expansions, as explained in Example (3.5.4)(a). One verifies immediately that the functions (5.1.2) satisfy the cocycle conditions and thus define, by gluing, an NC-manifold $P_{\text {left }}^{n}$.

The right projective space $P_{\text {right }}^{n}=\left(P^{n}, \mathcal{O}_{\text {right }}^{\mathrm{NC}}\right)$ is defined in a similar way, using the other order of division. One verifies immediately that $\mathcal{O}_{\text {right }}^{\mathrm{NC}}$ is anti-isomorphic, as a sheaf of rings, to $\mathcal{O}_{\text {left }}^{\mathrm{NC}}$.

Further, we construct a sheaf $\mathcal{O}_{\text {left }}^{\mathrm{NC}}(-1)$ of left $\mathcal{O}_{\text {left }}^{\mathrm{NC}}$-modules, locally free and of rank 1 , by using the 1-cocycle of the covering $\left\{U_{i}\right\}$ in $\left(\mathcal{O}_{\text {left }}^{N C}\right)^{*}$ given by

$$
\phi_{i_{j}}=t_{i} t_{j}^{-1}=x_{j}^{(i)}=\left(x_{i}^{(j)}\right)^{-1} \in \Gamma\left(U_{i} \cap U_{j},\left(\mathcal{O}_{\text {left }}^{\mathrm{NC}}\right)^{*}\right) .
$$

The dual sheaf $\mathcal{O}_{\text {left }}^{\mathrm{NC}}(+1)=\operatorname{Hom}\left(\mathcal{O}_{\text {left }}^{\mathrm{NC}}(-1), \mathcal{O}_{\text {left }}^{\mathrm{NC}}\right)$ is a sheaf of right $\mathcal{O}_{\text {left }}^{\mathrm{NC}}$-modules, locally free of rank 1. The sheaves $\mathcal{O}_{\text {left }}^{\mathrm{NC}}(m)$ for other $m$ do not make sense.

(5.1.3) Remark. Thus our construction of noncommutative projective spaces differs from the "noncommutative projectivization" of graded algebras as developed in [AZ] [Ros] [VW]. In our setting, a sheaf of left $\mathcal{O}$-modules on $P_{\text {left }}^{n}$ does not give rise to any graded module since the $\mathcal{O}(m), m \neq \pm 1,0$, are not defined. In fact, for a graded NC-milpotent algebra $R$ one can define a ringed space $\operatorname{Proj}(R)$ with the underlying topological space $\operatorname{Proj}\left(R_{a b}\right)$ by considering degree 0 elements in homogeneous localizations (cf. $\S 1$ ). Applying this construction to the algebras $\mathbf{C}\left\langle t_{0}, \ldots, t_{n}\right\rangle / F^{d+1}$ and passing to the inverse limit as $d \rightarrow \infty$, we get a sheaf of algebras $\mathcal{O}^{\text {big }}$ on $P^{n}$. This sheaf contains both $\mathcal{O}_{\text {left }}^{\mathrm{NC}}$ and $\mathcal{O}_{\text {right }}^{\mathrm{NC}}$ but is strictly bigger than any of them. In particular, $\left(P^{n}, \mathcal{O}^{\text {big }}\right)$ is not an NC-manifold.

(5.2) Grassmannians. Let $G(m, n)$ be the Grassmannian of $m$-dimensional subspaces in $\mathbf{C}^{n}$. The corresponding representable functor $h_{0}: \mathcal{C}$ om $\rightarrow$ Sets has the following explicit 
description. For a commutative algebra $\Lambda$ the set $h_{0}(\Lambda)$ consists of all submodules $V \subset \Lambda^{n}$ which are direct summands (hence projective) and have rank $\mathrm{m}$.

Now let us define a function $h: \mathcal{N} \rightarrow \mathcal{S}$ ets in a similar way: $h(\Lambda)$ is the set of left submodules $V \subset \Lambda^{n}$ which are direct summands and whose rank (as projective $\Lambda$-modules) is $m$.

(5.2.1) Theorem. The functor $h$ is formally smooth and commutes with arbitrary fiber products (2.3.3) in which at least one of the $p_{i}$ is surjective. Therefore (2.3.5) $h$ is represented by an NC-smooth thickening $G(m, n)_{\text {left }}^{\mathrm{NC}} \supset G(m, n)$.

Proof; To see the formal smoothness, it is enough to prove that $h\left(\Lambda^{\prime}\right) \rightarrow h(\Lambda)$ is surjective for any central extension

$$
0 \rightarrow I \rightarrow \Lambda^{\prime} \rightarrow \Lambda \rightarrow 0
$$

of NC-nilpotent algebras. Let us prove the following more general fact.

(5.2.2) Lemma. In the above situation let $P^{\prime}$ be a projective (left) $\Lambda^{\prime}$-module, $P=\Lambda \otimes P^{\prime}$ and $Q \subset P$ be a direct summand. Then the set of direct summands $Q^{\prime} \subset P^{\prime}$ such that $\Lambda \underset{\Lambda^{\prime}}{\otimes} Q^{\prime}=Q$, is a principal homogeneous space over

$$
I \underset{\Lambda_{a b}}{\otimes} \operatorname{Hom}_{\Lambda_{a b}}\left(Q_{a b}, P_{a b} / Q_{a b}\right) .
$$

Proof: It is enough to prove this under extra assumptions which can be satisfied by passing to localization. Then we would have that the direct summands $Q^{\prime}$ form a sheaf on the Zariski topology of $\operatorname{Spec}\left(\Lambda_{a b}\right)$ which is a torsor over the coherent sheaf corresponding to the $\Lambda_{a b}$-module specified above. Since $H^{1}$ of a coherent sheaf on an affine scheme vanishes, this torsor has a global section and thus the set of all its global sections is a principal homogeneous space as claimed.

Now, the extra assumptions we need, are:

(1) $P^{\prime}$ is free, $P^{\prime} \simeq\left(\Lambda^{\prime}\right)^{n}$

(2) $Q$ is free, $Q \simeq \Lambda^{m} \subset \Lambda^{n}$.

Let $e_{1}^{\prime}, \ldots, e_{n}^{\prime}$ be the standard basis of $\left(\Lambda^{\prime}\right)^{n}$, and $e_{1}, \ldots, e_{n}$ the corresponding basis of $\Lambda^{n}$. Since $Q=\left\langle e_{1}, \ldots, e_{m}\right\rangle$, an extension $Q^{\prime}$ of $Q$ is spanned by

$$
e_{i}^{\prime}+\sum_{j=m+1}^{n} b_{i j} e_{j}^{\prime}, \quad i=1, \ldots, m, \quad b_{i j} \in I
$$

So there is a bijection between all extensions of $Q$ and all matrices $B=\left\|b_{i j}\right\| \in \operatorname{Mat}_{l, m-l}(I)$. The set of such matrices can be written invariantly as the tensor product $I \otimes \operatorname{Hom}_{\Lambda_{a b}}\left(Q_{a b}, P_{a b} / Q_{a b}\right)$. Even though the constructed identification of the set of exten$\Lambda_{a b}$ sions with this tensor product is not canonical, the corresponding structure of a principal homogeneous space is, as it can be checked directly. This proves Lemma 5.1.2 and the formal smoothness of $h$. 
Let now $p_{i}: \Lambda_{i} \rightarrow \Lambda, i=1,2$, be given and at least one of the $p_{i}$ is surjective. In addition to the natural map

$$
j: h\left(\Lambda_{1} \underset{\Lambda}{\times} \Lambda_{2}\right) \rightarrow h\left(\Lambda_{1}\right) \underset{h(\Lambda)}{\times} h\left(\Lambda_{2}\right),
$$

let us define a map

$$
k: h\left(\Lambda_{1}\right) \underset{h(\Lambda)}{\times} h\left(\Lambda_{2}\right) \rightarrow h\left(\Lambda_{1} \underset{\Lambda}{\times} \Lambda_{2}\right)
$$

to associate to a pair $\left(V_{1} \subset \Lambda_{1}^{n}, V_{2} \subset \Lambda_{2}^{n}\right)$ with $\Lambda \underset{\Lambda_{1}}{\times} V_{1}=\Lambda \underset{\Lambda_{2}}{\times} V_{2}=V \subset \Lambda^{n}$, the submodule $\tilde{V}=V_{1} \underset{V}{\times} V_{2}$ in $\Lambda_{1} \times{ }_{\Lambda} \Lambda_{2}$. We need only to prove the lemma below. Then one verifies at once that $j$ and $k$ are mutually inverse.

(5.2.3) Lemma. $\tilde{V}$ is a direct summand of $\tilde{\Lambda}=\Lambda_{1} \underset{\Lambda}{\times} \Lambda_{2}$.

Proof: If $\Lambda$ is any ring and $V \subset \Lambda^{n}$ is a left submodule, then $\Lambda$ is a direct summand iff $\Lambda^{n} / V$ is projective. So the set of direct summands in $\Lambda^{n}$ identified with the set of surjections $s: \Lambda^{n} \rightarrow P$ with $P$ projective, modulo isomorphisms of projective modules.

Applying this to our particular situation, we have surjections $s_{i}: \Lambda_{i}^{n} \rightarrow P_{i}, s: \Lambda^{n} \rightarrow P$ and isomorphisms $\Lambda \underset{\Lambda_{i}}{\otimes} P_{i} \rightarrow P$ identifying $\Lambda \otimes s_{i}$ with $s$. In this case $\tilde{P}=P_{1} \underset{P}{\times} P_{2}$ is a projective $\tilde{\Lambda}$-module ([Mil], Th. 2.1) and we have a surjection $\tilde{s}: \tilde{\Lambda}^{n} \rightarrow \tilde{P}$ with kernel $\tilde{V}$. This proves the lemma and Theorem 5.2.1.

(5.2.4) Remarks. One can also construct $G(m, n)_{\text {left }}^{\mathrm{NC}}$ explicitly, by gluing $\left(\begin{array}{c}n \\ m\end{array}\right)$ copies of $A_{\mathrm{NC}}^{m(n-m)}$. For this, all we need is to mimic the standard formulas $[\mathrm{GH}]$ and interpret the entries of the inverse matrices involved, as commutator series, cf. (3.5.4)(c). This approach is closely related to the "noncommutative Grassmannian" construction of Gelfand and Retakh $[\mathrm{GR}]$. In fact, $G(m, n)_{\text {left }}^{\mathrm{NC}}$ can be seen as the formal completion of their construction along the commutative points.

(5.3) Flag varieties. Fix $1 \leq i_{1}-1<\ldots<i_{k} \leq n$ and let $F\left(i_{1}, \ldots, i_{k}, \mathbf{C}^{n}\right)$ be the variety of flags $V_{1} \subset \ldots \subset V_{k} \subset \mathbf{C}^{n}, \operatorname{dim}\left(V_{\nu}\right)=i_{\nu}$.

(5.3.1) Theorem. $F\left(i_{1}, \ldots, i_{k}, \mathbf{C}^{n}\right)$ has a natural $N C$-smooth thickening $F\left(i_{1}, \ldots, i_{k}, \mathbf{C}^{n}\right)_{\text {left }}^{\mathrm{NC}}$, and the action of $G L_{n}(\mathbf{C})$ extends to this thickening.

Proof: As in (5.2), we extend the functor represented by $F\left(i_{1}, \ldots, i_{k}, \mathbf{C}^{n}\right)$, to a functor $h: \mathcal{N} \rightarrow$ Sets with $h(\Lambda)$ being the set of all flags of left submodules $V_{1} \subset \ldots \subset V_{k} \subset \Lambda^{n}$ which are direct summands and such that the rank of $V_{\nu}$ is $i_{\nu}$. The fact that $h$ is formally smooth and commutes with fiber products such as in (5.2.1) is verified in a similar way, using Lemma 5.2.2 (plus induction) and Lemma 5.2.3.

(5.4) Moduli of vector bundles. Let $Z$ be a projective algebraic variety. For any variety $B$ let $\rho: B \times Z \rightarrow B$ denote the projection. 
(5.4.1) Definition. A versal family of vector bundles on $Z$ is a pair $(B, E)$ where $B$ is a smooth algebraic variety, $E$ a vector bundle on $B \times Z$ with the following properties:

(a) $R^{0} \rho_{*} \operatorname{End}(E)=\mathcal{O}_{B}$.

(b)The Kodaira-Spencer map $\kappa: T_{B} \rightarrow R^{1} \rho_{*} \operatorname{End}(E)$ is an isomorphism.

(c) $R^{2} \rho_{*} \operatorname{End}(E)=0$.

Many (etale) open charts of moduli spaces of stable vector bundles are bases of versal families.

(5.4.2) Theorem. If $(B, E)$ is a versal family of vector bundles, then $B$ admits a canonical NC-smooth thickening.

Proof: We construct a functor $h: \mathcal{N} \rightarrow$ Sets. Let $\Lambda$ be a NC-nilpotent algebra, $\Lambda_{a b}^{0}$ be the quotient of $\Lambda_{a b}$ by the ideal of nilpotent elements, $X_{a b}^{0}=\operatorname{Spec}\left(\Lambda_{a b}^{0}\right)$. The schemes $X_{a b}^{0}$ and $X_{a b}=\operatorname{Spec}(\Lambda)$ having the same underlying topological space, we can regard $X_{a b}^{0}$ as the underlying space for $\operatorname{Spec}(\Lambda)$, i.e., write $X=\operatorname{Spec}(\Lambda)=\left(X_{a b}^{0}, \mathcal{O}_{X}\right)$. Let $p_{1}, p_{2}$ be the projection of $X_{a b}^{0} \times Z$ to the factors. Denote by $X \times Z$ the NC-scheme with underlying space $X_{a b}^{0} \times Z$ and the sheaf of algebras $\mathcal{O}_{X \times Z}=p_{1}^{*} \mathcal{O}_{X} \otimes p_{2}^{*} \mathcal{O}_{Z}$. Its abelianization is $\mathcal{O}_{X_{a b} \times Z}$

Let $\mathcal{C}_{\Lambda}$ be the category whose objects are the following sets of data:

(1) A morphism of schemes $f: X_{a b}^{0} \rightarrow B$

(2) A locally free sheaf $\mathcal{E}$ of left $\mathcal{O}_{X \times Z}$-modules.

(3) An isomorphism $\varphi: \mathcal{O}_{X_{a b}^{0} \times Z} \otimes \mathcal{E} \stackrel{\sim}{\rightarrow}(f \times I d)^{*} E$.

A morphism $\left(f_{1}, \varepsilon_{1}, \varphi_{1}\right) \rightarrow\left(f_{2}, \varepsilon_{2}, \varphi_{2}\right)$ in $\mathcal{C}_{\Lambda}$ exists only if $f_{1}=f_{2}$ and consists of an isomorphism $\varepsilon_{1} \rightarrow \varepsilon_{2}$ commuting with the $\varphi_{2}$.

We define $h(\Lambda)$ to be the set of isomorphism classes of objects of $\mathcal{C}_{\Lambda}$. Our statement now will follow from (2.3.5) and the next proposition.

(5.4.3) Proposition. (a) The restriction of $h$ to Com coincides with $h_{B}$, the functor represented by $B$.

(b) $h$ is formally smooth.

(c) $h$ commutes with any fiber products (2.3.3) in which one of the $p_{i}$ is surjective.

Proof: We first concentrate on (b). It is enough to prove the following.

(5.4.4) Lemma. If

$$
0 \rightarrow I \rightarrow \Lambda^{\prime} \stackrel{p}{\rightarrow} \Lambda \rightarrow 0
$$

is a central extension of $N C$-nilpotent algebras and $[f, \varepsilon, \varphi] \in h(\Lambda)$, then $h(p)^{-1}[f, \varepsilon, \varphi]$ is a principal homogeneous space over $I \underset{\Lambda_{a b}}{\otimes} f^{*} R^{1} \rho_{*} \operatorname{End}(E)$.

Proof: As in the proof of (5.2.2), it is enough to work locally on $X_{a b}$, i.e., assume that $\Lambda_{a b}$ and $\Lambda$ are local rings. Then so is $\Lambda^{\prime}$. The set of isomorphism classes of locally free sheaves 
of left $\mathcal{O}_{X \times Z}$-modules of rank $r$ is in this case identified with $H^{1}\left(Z, G L_{r}\left(\mathcal{O}_{Z} \otimes \Lambda\right)\right)$, and similarly for $\Lambda^{\prime}$. We have an exact sequence

$$
1 \rightarrow \mathcal{O}_{Z} \times \operatorname{Mat}_{r}(I) \rightarrow G L_{r}\left(O_{Z} \otimes \Lambda^{\prime}\right) \stackrel{\tilde{p}}{\rightarrow} G L_{r}\left(\mathcal{O}_{Z} \otimes \Lambda\right) \rightarrow 1
$$

of sheaves of groups on $Z$. An element $[f, \mathcal{E}, \varphi] \in h(\Lambda)$ is represented by some $c \in$ $H^{1}\left(Z, G L_{r}\left(O_{Z} \otimes \Lambda\right)\right)$, while

$$
h(p)^{-1}[f, \mathcal{E}, \varphi] \cong \tilde{p}^{-1}(c) \subset H^{1}\left(Z, G L_{r}\left(\mathcal{O}_{Z} \otimes \Lambda^{\prime}\right)\right) .
$$

Our statement now follows from the general formalism of long exact sequences of nonAbelian cohomology, as recalled, e.g. in [Man], $\S(2.6 .8-9)$. Lemma is proved.

Thus we proved part (b) of (5.4.3). To prove part (a), note that by construction, $h=h_{B}$ on the category of commutative algebras without nilpotents. In addition, we have a natural transformation $u:\left.h_{B} \rightarrow h\right|_{\text {Com }}$, which sends a morphism $\tilde{f}: \operatorname{Spec}(\Lambda) \rightarrow B$ (with $\Lambda$ commutative) to the sheaf $(\tilde{f} \times I d)^{*} E$. We claim that $u$ is an isomorphism. To show this, it is enough to proceed by induction in the degree of nilpotency of the ideal $\operatorname{Ker}\left(\Lambda \rightarrow \Lambda_{0}\right)$, and consider a square-zero extension of commutative algebras, such as in (5.4.4) for which $u_{\Lambda}: \operatorname{Hom}(\operatorname{Spec}(\Lambda), B) \rightarrow h(\Lambda)$ is an isomorphism. Given $\tilde{f} \in h_{B}(\Lambda)$, the set $h_{B}(p)^{-1}(\tilde{f})$ is a principal homogeneous space over $I \underset{\Lambda}{\otimes} \tilde{f}^{*} T_{B}$, while by $(5.4 .4), h(p)^{-1}(\tilde{f})$ is a principal homogeneous space over $I \otimes R^{1} \rho_{*}(\operatorname{End}(E))$. Since the Kodaira-Spencer map $\kappa: T_{B} \rightarrow R_{1} \rho_{*}(\operatorname{End}(E))$ is an isomorphism by (5.4.1)(b), and $u_{\Lambda^{\prime}}$ is compatible with $\kappa$, it is a bijection.

Finally, we prove part (c) of (5.4.3). Given any diagram of algebras as in (2.3.3), we define the map

$$
k: h\left(\Lambda_{1}\right) \underset{h(\Lambda)}{\times} h\left(\Lambda_{2}\right) \rightarrow h\left(\Lambda_{12}\right)
$$

as follows. Given $f_{i}$ : $\operatorname{Spec}\left(\Lambda_{i, a b}^{0}\right) \rightarrow B$ coinciding on $\operatorname{Spec}\left(\Lambda_{12, a b}^{0}\right)$ and locally free sheaves $\varepsilon_{i}$ of $\mathcal{O}_{X_{i} \times Z}$-modules, isomorphic upon the pullback to $\mathcal{O}_{X_{12}} \times Z$, we define

$$
k\left(\left[f_{1}, \mathcal{E}_{1}, \varphi_{1}\right],\left[f_{2}, \mathcal{E}_{2}, \varphi_{2}\right]\right)=\left[f_{12}, \mathcal{E}_{12}, \varphi_{12}\right]
$$

where $f_{12}=f_{1} \times f_{f}, \mathcal{E}_{12}=\mathcal{E}_{1} \underset{\mathcal{E}}{\times \mathcal{E}_{2}}$, and $f: \operatorname{Spec}\left(\Lambda_{12, a b}^{0}\right) \rightarrow B, \mathcal{E}=\mathcal{O}_{X_{12} \times Z} \underset{\mathcal{O}_{X_{i}} \times Z}{\otimes} \mathcal{E}_{i}$ are the common restrictions of $f_{i}, \mathcal{E}_{i}$ on $\Lambda$. The sheaf $\mathcal{E}_{12}$ is locally free by Theorem 2.1 from [Mil] already used in the proof of (5.2.3). Having constructed $k$, it is clear that it is the inverse of the map $j$ from (2.3.4). So both are bijections.

This completes the proof of Proposition 5.4.3 and Theorem 5.4.2.

(5.4.6) Remark. The reason for all the examples considered in this section, to possess natural NC-thickenings, can be traced to the fact that he group $G L_{n}(\Lambda)$ can be defined for any associative and not necessarily commutative algebra $\Lambda$. This can be expressed by saying that the natural NC-thickening $G L_{n}(\mathbf{C})^{\mathrm{NC}} \supset G L_{n}(\mathbf{C})$ induced by the embedding $G L_{n}(\mathbf{C}) \subset A^{n^{2}} \subset A_{\mathrm{NC}}^{n^{2}}$, is a group object in the category of NC-manifolds. 


\section{References}

[AZ] M. Artin, J. J. Zhang, Noncommutative projective schemes, Adv. in Math. 109(1994), 228-287.

[BE] N. Blackburn, L. Evens, Schur multipliers of p-groups, J. Reine und Angew. Math. 309(1979), 100-113.

[Bry] J.-L. Brylinski, Loop spaces, characteristic classes and geometric quantization, Birkhäuser, Boston, 1993.

[Co] A. Connes, Noncommutative geometry, Academic Press, 1994.

[CQ] J. Cuntz, D. Quillen, Algebra extensions and nonsingularity, J. AMS, 8(1995), 251289.

[Eis] D. Eisenbud, Commutative algebra with a view towards algebraic geometry, SpringerVerlag, 1995.

[Ev] L. Evens, Terminal p-groups, Illinois J. Math. 12(1968), 682-699.

[Fe] R. Feynman, An operator calculus having applications in quantum electrodynamics, Phys. Rev. (2), 84(1951), 108-128.

[GH] P.Griffiths, J. Harris, Principles of algebraic geometry, J. Wiley, 1978.

[GK1] E. Getzler, M. Kapranov, Cyclic operads and cyclic homology, in "Geometry, topology and physics for R. Bott" (S. T. Yau, Ed.), P. 167-201, International Press, Cambridge MA, 1995.

[GK2] E. Getzler, M. Kapranov, Modular operads, Compositio Math. 110(1998), 65-126.

[GR] I. M. Gelfand, V. S. Retakh, Quasideterminants I, preprint alg-geom/9705026, to appear in Selecta Math.

[HH] J. W. Helton, R. E. Howe, Traces of commutators of integral operators, Acta Math. 135(1975), 271-305.

[HS] V. S. Hinich, V. V. Schechtman, On homotopy limit of homotopy algebras, in " $K$ theory, arithmetic and geometry" (Y. I. Manin Ed.), p. 240-264, Lecture Notes in Math. 1289, Springer-Verlag, 1987.

[Kap] M. Kapranov, Rozansky-Witten invariants in Atiyah classes, preprint alg-geom \# 9704009, to appear in Compositio Math.

$[\mathrm{KM}]$ M. V. Karasev, V. P. Maslov, Nonlinear Poisson brackets, geometry and quantization, Amer. Math. Soc. 1993.

[Ko] M. Kontsevich, Formal non-commutative symplectic geometry, in: "Gelfand Mathematical Seminars 1990-92" (L.Corwin, I. Gelfand, J. Lepowsky Eds.), 173-187, Birkhauser, Boston, 1993.

[L] J.-L. Loday, Cyclic homology, Springer-Verlag, 1992. 
[MKS] W. Magnus, A. Karrass, D. Solitar, Combinatorial group theory, Dover Publ. 1976. [Mac] I. Macdonald, Symmetric functions and Hall polynomials, Oxford, Clarendon Press, 1995.

[Man] Y. I. Manin, Gauge field theory and complex geometry, Springer-Verlag, 1991.

[Mil] J. Milnor, Introduction to algebraic K-theory, Princeton Univ. Press, 1971.

[Mas] V. P. Maslov, Operational methods, Mir Publ. Moscow 1968.

[Ros] A. L. Rosenberg, Noncommutative algebraic geometry and representations of quantum groups, Kluwer Publ. 1995.

[Sche] W. Schelter, Smooth algebras, J. of Algebra, 103(1986), 677-685.

[Schl] M. Schlessinger, Functors on Artin rings, Trans. AMS, 130(1968), 208-222.

[Sig] S. Sigg, Laplacian and homology of free two-step nilpotent Lie algebras, J. Algebra, 185(1996), 144-161.

[Sta] J. D. Stasheff, Homotopy associativity of $H$-spaces I, II, Trans. AMS, 108(1963), 275-292.

[Ste] B. Steinstrom, Rings of quotients, Springer-Verlag 1975.

[VV] F. Van Oystaeyen, A. H. Verschoren, Non-commutative algebraic geometry, Lecture Notes in Math. 887, Springer-Verlag 1981.

[VW] F. Van Oystaeyen, L. Willaert, Cohomology of schematic algebras, J. Algebra, 185(1996), 74-84.

Author's address: Department of mathematics, Northwestern University, Evanston IL 60208 USA, email:

kapranov@math.nwu.edu 